\title{
ARCHITECTURES FOR FUTURE MULTIGIGABIT LIGHTWAVE NETWORKS
}

\section{William E. Burr}

U.S. DEPARTMENT OF COMMERCE Natlonal Instltute of Standards and Technology

Natlonal Computer Systems Laboratory Galthersburg, MD 20899

U.S. DEPARTMENT OF COMMERCE Robert A. Mosbacher, Secretary Lee Mercer, Deputy Under Secretary for Technology

NATIONAL INSTTUTE OF STANDARDS AND TECHNOLOGY

John W. Lyons, Director

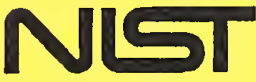





\section{ARCHITECTURES FOR FUTURE MULTIGIGABIT LIGHTWAVE NETWORKS}

\section{William E. Burr}

U.S. DEPARTMENT OF COMMERCE Natlonal Instlitute of Standards and Tochnology

Natlonal Computer Systems Laboratory Galthersburg, MD 20899

Fobruary 1990

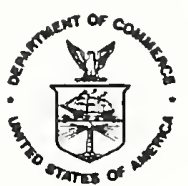

U.S. DEPARTMENT OF COMMERCE Robert A. Mosbacher, Secretary Lee Mercer, Deputy Under Secretary for Technology

NATIONAL INSTITUTE OF STANDARDS AND TECHNOLOGY

John W. Lyons, Director 



\title{
Architectures for Future Multigigabit Lightwave Networks
}

\author{
William E. Burr
}





\section{Summary}

This report examines possible future fiber optic network architectures for voice, video and data applications. The telephone industry is planning to introduce Broadband ISDN (BISDN) for these applications sometime after 1995. B-ISDN will be based on fast packet switching of rather small (48-byte) packets. The packet switches themselves will most probably be electronic, and of great complexity. They will take advantage of the ability of mature semiconductor electronic technology to build very complex, regular, repetitive structures. Packet switching provides a flexible, efficient means of sharing bandwidth limited communications resources. Fiber optic technology, however, offers another, revolutionary, alternative, the Passive Optical Network (PON), based on the realization that the inherent bandwidth of the fiber is immense, and using frequency division multiplexing as the vehicle for exploiting this. The PON has significant advantages over centralized packet switching in some applications (particularly video distribution), is much simpler, facilitates the addition of new originally unanticipated services, can be begun and extended in small increments, and requires much less initial investment in the network. However the PON depends upon the commercial development of several components, such as tunable lasers and coherent receivers, which have so far been proven only in the laboratory environment. 


\section{Introduction}

The immense bandwidth of optical fiber, although so far barely exploited, has already caused optical fiber to take over all new long haul telecommunications applications. Whereas a decade ago there was no fiber in the distribution network, today virtually all new long distance terrestrial telecommunications circuits are fiber optic, and the replacement of most long distance electrical telecommunions circuits seems certain. So far, however, the use of fiber has not been revolutionary, it has simply been used as a substitute medium for conventional communications circuits (to the extent of emulating even the multiplexing structure of the electrical circuits for which it is substituted). It is likely, however, that the full exploitation of fiber optics will cause fundamental changes in the basic architecture of telecommunications.

It is commonly claimed that communications technology oscillates between a situation where switching is expensive and transmission is comparatively inexpensive, and the reverse. In the 1950s, the use of microwaves made transmission comparatively inexpensive, but switching with relays or vacuum tubes was expensive. By the 1970s, the development of semiconductor ICs had allowed the development of powerful digital circuit switches and allowed general purpose computers to serve as store and forward packet switches (in the ARPA Net, for example), but data transmission was comparatively slow and expensive, favoring the development of computationally complex computer data transmissions protocols, such as TCP/IP. Now, in the late 1980s, with high speed fiber optic links, communications speeds have already outstripped the speed of switches yet, the bandwidth potential of current optical fiber is many orders of magnitude greater than has so far been exploited.

There is now much discussion of the possibility of integrating high bandwidth services, including voice, data and video, by fast transmission of short (48-byte) packets. This is the principal mechanism proposed for the future Broadband Integrated Services Digital Network (B-ISDN). Packet switching is an excellent mechanism for efficient use of a limited bandwidth channel with a rapidly changing load. It is far from clear, however, that it is the best or most natural way to design future networks, because we appear to be entering an era when media bandwidth is nearly unlimited, but switching, not transmission, is comparatively more expensive and difficult.

This paper explores the architectures which might be used in future high bandwidth integrated services networks. The term network architecture is used here to be the network topology, transmission and modulation technology and the basic network access mechanisms and is therefore covers only the lower three layers of the seven layer Open Systems Interconnection (OSI) reference model. Above layer three there should be little effect on higher layer protocols. Three basic types of services are contemplated in integrated networks: voice, computer data and video. 


\section{Applications}

Three distinct applications for future high bandwidth networks are considered in this report: voice, computer data and video. The primary emphasis will be on local and metropolitan area distribution, rather than long haul distribution. There are three reasons for this: (1) fiber has so far made little penetration in this more cost sensitive area, (2) there is more investment required for local distribution than for long distance distribution, since about $90 \%$ of all US telephone circuits are local loops [118] and (3) the long distance communications needs for the near future can probably be met by incremental improvements in fiber optic transmission technology rather than architectural innovation, until changes in the local distribution fabric and load fundamentally change the nature of the long distance load.

\section{Voice}

The present telephone network is driven by the voice application. A significant part of the current plant, including nearly all telephone instruments, and many older switches and trunks, are analog. Switching and interoffice trunking are increasingly being converted to digital services. The digital telephone system is slaved to an $8 \mathrm{kHz}$ clock. Telephone signals are considered to be band limited to below $4 \mathrm{kHz}$, so an $8 \mathrm{kHz}$ sampling rate is used. An 8 bit digital sample provides an adequate signal to noise ratio, giving a basic data rate of 64,000 bits/second. This is almost the only common thread in the present worldwide digital telephone system, since Europe and North America have different carrier multiplexing structures above this rate. In the present North American System the two principal multiplexed carriers are DS-1, which carries 24 basic voice circuits giving a rate of $1.536 \mathrm{Mbit} / \mathrm{s}$, and DS-3, which carries 28 DS1 channels or 672 voice channels, yielding a data payload of 43.008 Mbit/s.

Isochronism, that is the delivery of data at a constant, unvarying rate, is an important property of the current telephone network. It would, in principle, be possible to build a system which allowed some short term variation in data transfer rate (which would be necessary in many packet voice technologies) by using buffers to smooth the arrival of data. Such buffers would, however, introduce delay. The delay is important because most existing analog instruments are connected to the network through a single, imperfectly terminated, bidirectional copper wire pair. Reflected signals are transmitted back to the sender, and if delays exceed a few ms, they are heard as an annoying echo. Echo cancellation circuits are now available to eliminate this problem, but there is a vast installed plant in which echo is and will remain a problem for some time.

The Integrated Services Digital Network (ISDN) is an attempt to introduce end to end digital telephone and data service to the worldwide telephone network. The fundamental ISDN service is two duplex $64 \mathrm{kbit} / \mathrm{s}$ voice or data circuits (called "B" channels) plus a single $16 \mathrm{kbit} / \mathrm{s}$ packet channel (called a " $D$ " channel) used primarily for call setup and establishment (called "signalling" in ISDN parlance). The ISDN paradigm is an integrated voice and data terminal, on which it will be possible to both transfer data at $64 \mathrm{kbit} / \mathrm{s}$ and simultaneously have a telephone conversation. Above the $2 \mathrm{~B}+\mathrm{D} 144 \mathrm{kbit} / \mathrm{s}$ service North America and Europe diverge, with the North American "primary" rate service being $23 \mathrm{~B}+\mathrm{D}$ channels, all $64 \mathrm{kbit} / \mathrm{s}$, for a DS- 1 compatible total of $1.536 \mathrm{Mbit} / \mathrm{s}$. The European primary rate has a total of $30 \mathrm{~B}+1 \mathrm{D} 64 \mathrm{kbit} / \mathrm{s}$ channels for a total rate of $1.984 \mathrm{Mbit} / \mathrm{s}$. Although ISDN has been under development since the mid 1970s, there are now only isolated islands of ISDN in various cities and trials around the world. Present incompatibilities between switches of various vendors restrict the interconnection of these islands, and far 
less than $1 \%$ of the worlds telephone instruments are ISDN instruments. ISDN capable switches are available from several major switch vendors, but interswitch compatibility problems plague the transparency of a worldwide ISDN network.

It is important not to confuse the intrinsic requirements of voice service with the artifacts of a comparatively old telephone network. For example, with suitable coding, easily provided on an inexpensive IC, telephone quality voice can be provided at rates of 16-kbit/s or less. With appropriate buffering in instruments or switches, and with echo cancellation for old analog instruments, if they are retained, considerable jitter in voice packet arrival could be accommodated, and strict isochronism made unnecessary. One important difference between voice and computer data remains: high error rates are easily tolerated in highly redundant voice communications (although error rates become more of a concern as bandwidth is compressed), while very low error rates are required for computer data transmission.

\section{Computer Data Services}

It is dangerous to generalize about computer data services, since many different applications exist, each with its own requirements. One general characteristic, however, is that error rates are expected to be very low, at least in high performance networks. In the FDDI LAN, the normal maximum link error rate goal is $10^{-12}$, while the worst case allowed is $2.5 \times 10^{-10}$. The maximum end to end network bit error rate goal is $10^{-9}$ [29], resulting from the sum of all the link error rates. One of the principal designers of FDDI, however, thinks that this is too high and argues for $10^{-15}, 2.5 \times 10^{-13}$ and $10^{-12}$, respectively [38].

In addition, computer networks such as FDDI now routinely employ error detecting codes, which detect all one or two bit errors in packets, and will pass as good fewer than one packet in $10^{9}$ packets of completely random data [27]. Indeed, with suitable error detecting and error correcting codes, computer communications can reach acceptable levels of data integrity, even with very noisy channels, but there are considerable cost and performance penalties for such noisy channels. Current computer network protocols, designed to operate reliably over noisy channels are often notoriously slow when operated over a reliable, high bandwidth LAN.

While much data in computer systems is quite noisy before it enters the system, local computer systems have always been designed to operate without adding to this noise while storing and operating on data. All data is treated as if it were a program, the corruption of a single bit of which can be catastrophic. A supercomputer, consisting of many millions of gates, is expected to be able to run a complex simulation, requiring days for its completion and immense numbers of calculations. Given the same input data and program, the computer is also expected to generate exactly the same result, to the last bit, every time it is run.

As networks extend the scope of the computer systems the same principle applies; this has been increasingly achieved in local networks and will apply to long distance networks as well. Computer system designers want channels whose noise is too low to measure, and then they check every packet anyhow. The goal is no undetected errors, ever, and computer systems come increasingly close to this. In ideal computer systems, no errors originate in the data transmission, storage or computation, but if they do, they are detected and, if possible, corrected. All errors can then be ascribed to data entry errors and programming errors. The first requirement of computer data applications is very low channel error rates 
even for very long distance communication. This goal can be met by a variety of means, but if high performance is also a goal, then the "raw" physical level error rate must be low.

Beyond this, applications diverge widely in their requirements. For example, many transaction applications are limited by the speed at which a human can read or type text. An example is an airline reservation system. Such systems have rather low bandwidth requirements (the $64 \mathrm{kbit} / \mathrm{s}$ ISDN rate is probably overkill) and moderate delay requirements. In many cases transaction applications are datagram oriented or connectionless, that is they consist of one or two short exchanges of data in each direction and the transaction is over. The next transaction may be to an entirely different destination. Most transactions require either a response or acknowledgement of the receipt of the transaction. In the future transactions may involve images, for example the image of a person seeking entry into a secure area. When images are involved, the data required will typically increase by orders of magnitude.

Computer mail, messages and facsimile transfers will usually involve more data than transaction systems, enough, in many cases, to warrant a connection at some level. They can tolerate comparatively long delays, however, from seconds to hours, depending on the service. Bulk transfers of entire large files are a special, but important, class of mail which transfer larger volumes of data.

Direct on-line access to mass storage is probably the most demanding requirement, involving both the transfer of large amounts of data and short latencies. Delay in the network should be held to not more than the same general order as latencies involved in accessing the data in electromechanical storage devices $(10$ to $30 \mathrm{~ms})$. If this is done, remote storage can be accessed as if it were local, with little performance penalty. Network storage servers are now common in LANs, but, with suitable networks, servers for metropolitan areas and even entire regions of the country are possible with only moderated decreases in performance due to speed of light delays. The future corporate computer becomes a collection many loosely coupled processors and storage servers, spread over a plant, campus, metropolitan area, region or continent, with data access latencies, but not data rates, limited by speed of light delays. Since files are typically opened, processed for a while, then closed, even within a single computer, a connection oriented service fits.

Real time and process control applications present their own peculiar requirements, largely guarantees about the maximum delay in accessing the network. The time constants of many applications may confine them to very localized environments, but others (for example load management of a large power grid) are inherently widely distributed.

\section{Video}

Video is often considered to be the critical element in bringing fiber all the way to subscribers and to completely dominate all other potential high bandwidth subscriber applications [71], [75]. That is, it is the only service seen as having a high interest in the residential market, for which the high bandwidths of fiber are needed. At the moment, however, while fiber is used for trunking in some Cable Access Television (CATV) systems, it has made no impression whatever in direct subscriber applications. This is an area fraught with uncertainties, including the expectation that present low resolution television will be supplanted soon by "High Definition Television" (HDTV), as well as the hope that HDTV is the primary application which will drive the installation of fiber for local distribution. 
The exact nature of the HDTV service is uncertain. There will probably be several levels of service introduced in various parts of the world, including "enhanced" services which improve upon the present NTSC system (used in the US and 34 other countries), while preserving some level of compatibility with current receivers, as well as studio quality true HDTV.

While HDTV systems with 1050, 1125 and 1250 lines have been proposed [86], one proposal made to the International Radio Consultative Committee (CCIR) in 1986, based upon a system developed by the Japan Broadcasting Company (NHK), and described in the CCIR "Green Book" that year, is probably becoming the basis for a de facto standard studio system. It is already in use in studios in several countries [39], [44], [70]. A final decision should be made by CCIR in 1990. It is a 1125 line per frame, 60 fields per second, 2:1 interlaced system with a 16:9 aspect ratio. It has 1035 active horizontal lines and 1920 luminance samples per horizontal line. As originally proposed, it was a 4:2:2 system, that is the ratio of the luminance was twice that of the two color difference signals 1 . The Society for Motion Picture and Television Engineers (SMPTE) has argued for a 4:4:4 standard for studio use [44], and a 4:1:1 signal might be considered adequate for HDTV distribution to residences.

A rival European proposal advocates 1:1 progressive scanning of 1250 lines with 1152 active lines, a 16:9 aspect ratio, a $50 \mathrm{~Hz}$ field frequency and 1920 samples per line [70]. We will consider the NHK proposal, which the United states supports and for which commercial equipment exists, to be the likely standard in North America.

Consider the transmission bandwidth requirements of video. In North America and Japan the broadcast TV system is NTSC. NTSC uses 525 lines, interlaced 2:1, with 60 fields per second. The 2:1 interlace means each scan field includes only half the lines, so only 30 complete images are sent each second. The interlace cuts the bandwidth in half, at the expense of some image resolution and interline flicker (30 full uninterlaced fields per second would result in very perceptible large field flicker). The broadcast standard specifies a $4.5 \mathrm{MHz}$ bandwidth set at $6 \mathrm{MHz}$ intervals. The 68 broadcast channels require a bandwidth of $408 \mathrm{MHz}$. Some cable systems provide for as many as 120 channels with a bandwidth requirement of $720 \mathrm{MHz}$. To date, commercial analog base band fiber based TV links have been limited to about 30 channels by the nonlinearities of the linear devices available and these have been used to some extent as trunks in CATV distribution systems.

To avoid the degradation of signal quality inherent in many successive analog repeaters, long distance transmission of video is often digital. Transmission of high quality composite NTSC video is conventionally done with two DS-3 $43.008 \mathrm{Mbit} / \mathrm{s}$ channels [93]. The channel is band limited to $4.5 \mathrm{Mhz}$. The color subcarrier frequency is approximately $3.58 \mathrm{MHz}$. To prevent aliasing, it is necessary to filter the signal above $4.5 \mathrm{MHz}$ and to oversample at a

1 Video signals originate in the camera and are processed in studios as three separate red, blue and green ( $R B G$ ) signals. To save bandwidth and to produce a signal which is compatible with monochrome sets, video is usually transmitted as a composite signal with a luminance signal $(Y=R+B+G)$ and two color difference signals (R-Y and B-Y). Since the eye sees much finer luminance detail than color detail, the bandwidth of the two color difference signals is usually filtered to reduce it to less than the luminance bandwidth. 
rate greater than the filtered bandwidth. It is convenient to use three times the subcarrier frequency, about $10.7 \mathrm{MHz}$, as the sampling frequency. With an 8 bit sample (sufficient to provide about a $56 \mathrm{~dB}$ signal to noise ratio), this results in a data rate of $85.6 \mathrm{Mbit} / \mathrm{s}$ or almost twice the DS-3 rate.

Terrestrial broadcasting spectrum utilization forces AM video transmission, which minimizes the bandwidth used. If optical fiber were used for analog transmission, these bandwidth constraints would not prevail and frequency modulation would offer several advantages. A comparison of the receiver bandwidths for equivalent quality NTSC digital FSK video and FM analog video (often used for satellite broadcasting which, as with fiber, tends to be power rather than bandwidth limited) estimates these to be $120 \mathrm{MHz}$ and $30 \mathrm{MHz}$, respectively [75].

The two systems used in Europe (PAL and SECAM) use 625 lines, interlaced 2:1, with 50 fields per second. CCIR Recommendation 601 for the studio coding of video for PAL, SECAM or NTSC uses 4:2:2 components with a sampling rate $13.5 \mathrm{MHz}$ for luminance and 6.75 $\mathrm{MHz}$ for the two color difference signals, yielding (with an 8-bit sample), a data rate of $216 \mathrm{M} / \mathrm{Bit} / \mathrm{s}$. RGB video coded in this way is easy to convert between the three systems, and recommendation 601 is widely accepted as a studio standard for the three present broadcast systems.

What are the bandwidth requirements of HDTV? Ideally the full studio quality would be delivered direct to residences. The HDTV studio system described in the Green Book requires a luminance bandwidth of $30 \mathrm{MHz}$, and the Advanced Television Standards Committee (ATSC) has recommended a sampling frequency of $74.25 \mathrm{MHz}$, yielding, a brute force data rate for luminance of $592 \mathrm{Mbit} / \mathrm{s}$, and, assuming a 4:2:2 system, a total rate of 1.184 Gbit/s. While this can be reduced somewhat by not digitizing anything but active lines (assuming storage in the receiver), the overall rate is, in round numbers, $1 \mathrm{Gbit} / \mathrm{s}$.

The coding and compression of HDTV for use in B-ISDN has been studied intensively, and rates from 30 to $135 \mathrm{Mbit} / \mathrm{s}$, compatible with the proposed B-ISDN $\mathrm{H}_{\mathbf{n}}$ carrier structure, are capable of carrying good HDTV signals, with suitable compression. As the rates decrease, however, the cost of the codec goes up, so the 135 megabit rate, compatible with the B-ISDN H4, rate is deemed to be the best compromise [89], [69], [32]. Both temporal and spatial compression are required, to produce the rough equivalent of the studio quality HDTV at the $\mathrm{H}_{4}$ rate.

The B-ISDN plan for direct HDTV to the home is to provide one or two switched 135 Mbit/s HDTV channels at the home. Particular channels would be selected from the choices available at the local switch. One consequence of the considerable compression required for $135 \mathrm{Mbit} / \mathrm{s} \mathrm{HDTV}$ is that the effect of transmission errors is magnified and very low bit error rates (on the order of $10^{-10}$ to $10^{-11}$ ) are required [69].

Several points about B-ISDN as a home video distribution medium should be obvious. Distribution of video to homes is basically a one way service. Only one or two channels at a time through B-ISDN is inferior to present cable services which may offer 30 or more, and a broadcast service theoretically offering 68 channels; since many homes may contain more than one or two sets or recorders, and some sets can now display 2 channels at once.

In the United States existing private broadcast and cable interests are very powerful and it is far from certain that regulated public telephone carriers will ever be allowed to compete 
with cable and broadcast interests to distribute video to residences, or, if they are, that they would be successful, irrespective of the transmission technology. Vertical integration of programming and distribution may be a more powerful competitive combination than integration of voice and data distribution with residential video. The situation may be very different in this respect elsewhere in the world, where private broadcasting is more the exception than the rule, and governments and public telephone carriers may be able to impose solutions on broadcasters which would not be possible in the United States.

HDTV is offered as an incentive for moving to B-ISDN, yet the basic market appeal of HDTV is unproved. At normal viewing distances on normal size (27 in diagonal or less) screens there is no dramatic visual difference between HDTV and present NTSC video for normal TV programming. Much larger high resolution displays are required to provide movie theater like visual impact [92]. Large screen, high resolution TVs, with fairly complex digital decoders for compressed HDTV video (if the B-ISDN $135 \mathrm{Mbit} / \mathrm{s}$ service is used for distribution) will be required; they will surely be quite expensive, at least initially. The significant quality advantage of laser disks over video tape has not prevented tape from dominating the home video market, indicating that the viewing public does not consider picture quality to be as important as the ability to record. The market appeal of HDTV depends somewhat upon its price, and the programming available, and it is very hard to predict at what rate it is likely to develop.

If B-ISDN subscriber HDTV service is offered, it will have to compete with CATV HDTV alternatives, very likely fiber based, at least to the final stage of distribution, terrestrial broadcast HDTV, direct satellite broadcast TV, and tape and disk based publishing of video material. These may well be analog services, but analog distribution to the home should make for less expensive sets, and quality can be quite high. Long distance terrestrial transmission can be accomplished by conversion to digital form, with reconversion to analog for local distribution, as it is now.

The greatest barrier to the introduction of a one or two channel at a time B-ISDN switched HDTV system is simply the huge present investment in NTSC. There are about 200 million NTSC receivers in 32 countries [26], millions of video recorders and massive investments in NTSC terrestrial broadcast and cable distribution systems. In the US it will be difficult to launch any new video service which does not effectively access this installed base. Of course, digital HDTV to NTSC decoders could be installed, but this would be an expensive way to provide 1 or 2 channels (at a time) of video, which would not necessarily be higher in quality than available over the air or on cable. Moreover NTSC will be a moving target since there are many proposals for enhancing the quality of NTSC. These proposals are compatible with the present receivers, yet offer enhanced receivers better picture quality, which may approach that of HDTV [26], [98], [51], [115], [45]. These can be broadcast over the air or from satellites, or distributed by conventional CATV systems. Any improved North American video distribution system must play to and service the installed base of NTSC receivers at least as well as the present system, or it will never be able to get started in the face of the immense market they represent. Improved systems in the United States must support NTSC.

It seems unlikely that HDTV will provide the economic basis for B-ISDN service to the home in North America. Nevertheless, there may be a considerable public network market for such applications as video conferencing, long distance video transmission, and monitoring of video cameras for security, traffic control and similar applications, to which B-ISDN might be more applicable. Many of these may not require HDTV quality. Other alterna- 
tives for broadcasting a number of channels in a variety of formats (including NTSC), over a very high bandwidth fiber optic equivalent of present CATV services, appear more promising as a means of realizing HDTV. They would more directly take advantage of the inherent strengths of optical fiber, and would provide a better justification for running fiber to residences. 


\section{The Technology}

In this section we will consider present fiber optic technology and its likely development. In the past decade we have gone through roughly three generations of optical fiber technology. The first generation, used multimode fibers operating at the $830 \mathrm{~nm}$ wavelength. It was followed by a second generation, using single mode fibers at the $1300 \mathrm{~nm}$ wavelength, and a third generation, for long distance applications, using single mode fiber at $1550 \mathrm{~nm}$. Each has represented a significant improvement in the bandwidth $\mathbf{x}$ distance product obtained. All were analogous, however to spark gap radio transmitters, using direct detection of baseband signals. Now we are poised for a fourth generation, which will use coherent detection and other wavelength dependent techniques to enormously increase the bandwidth of optical fiber. This, in a sense, is unleashing the genie already in the bottle, because the technology is largely applicable to the current installed base of single mode fiber, whose inherent bandwidth has only barely been exploited. The change will ultimately be as profound as the change from discrete transistors to VLSI integrated circuits. The several orders of magnitude improvement available in fiber bandwidth will permit truly revolutionary, rather than evolutionary, changes in communications networks.

\section{Present Use of Optical Fiber}

Until recently the major use of optical fiber has been as a substitute for conventional coaxial cable electrical carriers to increase the span between repeaters. In North America fiber has been widely substituted for coaxial cable in DS-3 with a payload rate of 43.008 Mbit/s. A single, or several multiplexed DS-3 channels, are carried on a single fiber pair. These have been non-standard links, where the repeaters and terminations on the ends are furnished by a single supplier, and interconnections across carrier boundaries, or between circuits developed by different suppliers, has been by conversion to the DS-3 electrical standard and back to fiber optics.

This is now expected to change rapidly with the development and expected rapid deployment of the Synchronous Optical NETwork (SONET) standard [8], [12]. SONET will be an important international standard for an optical carrier network. SONET will provide a variety of rates at multiples of $51.84 \mathrm{Mbit} / \mathrm{s}$, eventually extending up to at least 2488.32 $\mathrm{Mbit} / \mathrm{s}$, with early deployment of circuits at 155.52 and $622.08 \mathrm{Mbit} / \mathrm{s}$. The SONET frame structure is illustrated in figure 1. In addition to an optical carrier interface SONET defines a time division multiplexing structure for carrying virtually all current telephony circuits.

So far optical fiber has seen limited commercial use in computer Local Area Networks, however the development of the Fiber Distributed Data Interface (FDDI) standard will rapidly change this. FDDI is being supported by virtually all computer systems and LAN vendors worldwide. It provides a standard $100 \mathrm{Mbit} / \mathrm{s}$ token ring LAN and allows the interconnection of up to 500 stations through up to $200 \mathrm{~km}$ of fiber. FDDI provides for both multimode fiber with LED sources (for lower cost \& distances up to $2 \mathrm{~km}$ ) and single mode fiber with laser sources (for distances of up to $60 \mathrm{~km}$ ). By the mid 1990's, FDDI networks should be ubiquitous in LAN applications requiring high bandwidth, such as backbone networks connecting slower electrical LANs and as a direct network for engineering workstations and graphic application. By the turn of the century, FDDI will probably be a common office network, as office PCs become at least as powerful as present engineering workstations and as computer image processing becomes a routine part of the "paperless office." 


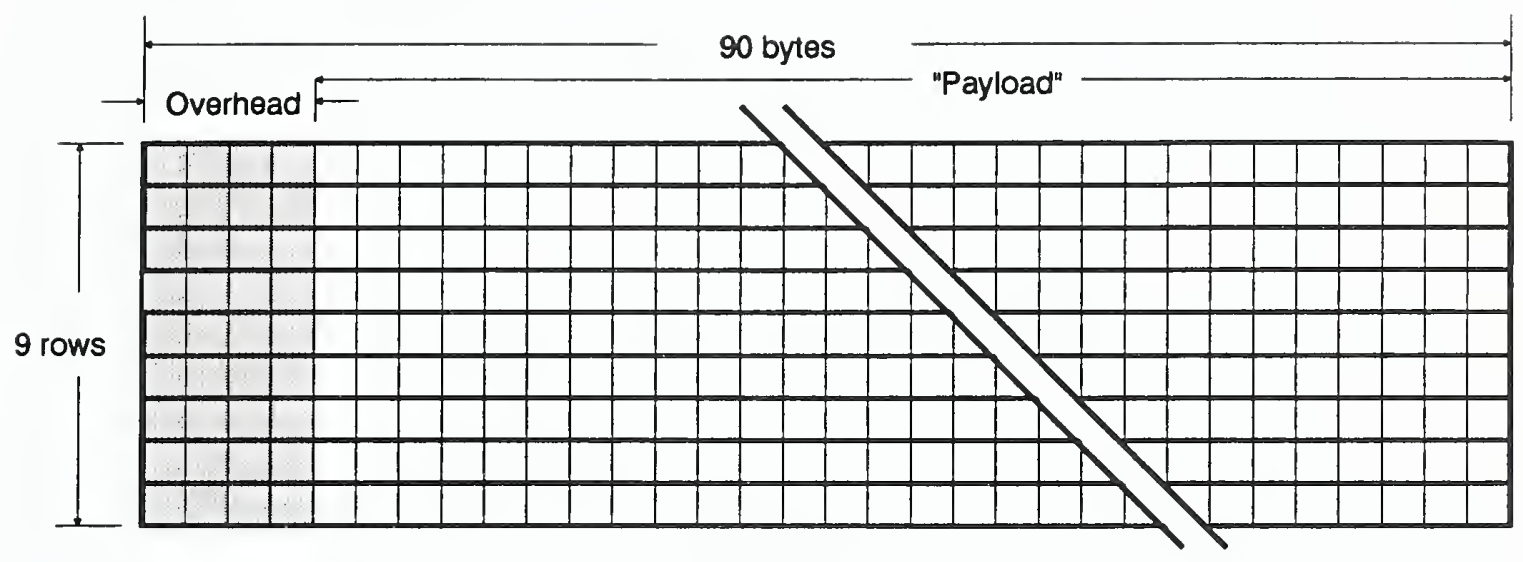

Figure 1 - SONET Frame Structure

\section{Media}

It appears likely that the medium for high bandwidth local and metropolitan area networks in the next two decades will continue to be single mode $\mathrm{Ge}$ doped $\mathrm{SiO}_{2}$ glass fiber. In 1970 Corning showed that $\mathrm{SiO}_{2}$ glass fibers could have a loss as low as $20 \mathrm{~dB} / \mathrm{km}$ [11], a landmark often taken to be the first demonstration of the practicality of fiber for data transmission. In a surprisingly short period $\mathrm{SiO}_{2}$ fiber has become a mature product, widely used in long haul telecommunications. It is already manufactured in large quantity at low cost, with properties which are, in many respects, very nearly as good as is theoretically possible. Current single mode fiber has an intrinsic bandwidth which current transmitter and receiver components barely use. The fundamental limitation on loss in this medium is Rayleigh scattering, which varies inversely with the forth power of wavelength, thus there is a considerable advantage to using as long a wavelength as possible [42]. At sufficiently low energies, however, the photons interact with the thermal vibrations of the glass causing infrared absorption, which rises rapidly as wavelengths exceed about $1500 \mathrm{~nm}$. This limits the minimum loss of $\mathrm{SiO}_{2}$ to a little less than $.2 \mathrm{~dB} / \mathrm{km}$ at around $1500 \mathrm{~nm}$. At shorter wavelengths absorption from $\left(\mathrm{OH}^{-}\right)$ions, which are very difficult to remove entirely from the glass, cause absorption bands or "water peaks" at around $1370 \mathrm{~nm}, 1230 \mathrm{~nm}$ and $950 \mathrm{~nm}$. Fiber is therefore spoken as having three "windows" at about $850 \mathrm{~nm}, 1300 \mathrm{~nm}$ and $1500 \mathrm{~nm}$, which are separated by $\left(\mathrm{OH}^{*}\right)$ absorption bands and bounded by infrared absorption above about $1550 \mathrm{~nm}$ and rapidly increasing Rayleigh scattering below $800 \mathrm{~nm}$. The loss curve of typical fiber is illustrated in figure 2 . Significantly lower attenuations are possible only by going to another material, with a longer wavelength before infrared absorption becomes significant.

The inherent bandwidth of single mode fiber is very high, more than $10,000 \mathrm{GHz}$ in the $1500 \mathrm{~nm}$ region alone (depending upon the cut-off point for allowable loss per $\mathrm{km}$ ) and the total bandwidth in the 1300 and $1500 \mathrm{~nm}$ regions is more than $30,000 \mathrm{GHz}$. For com- 


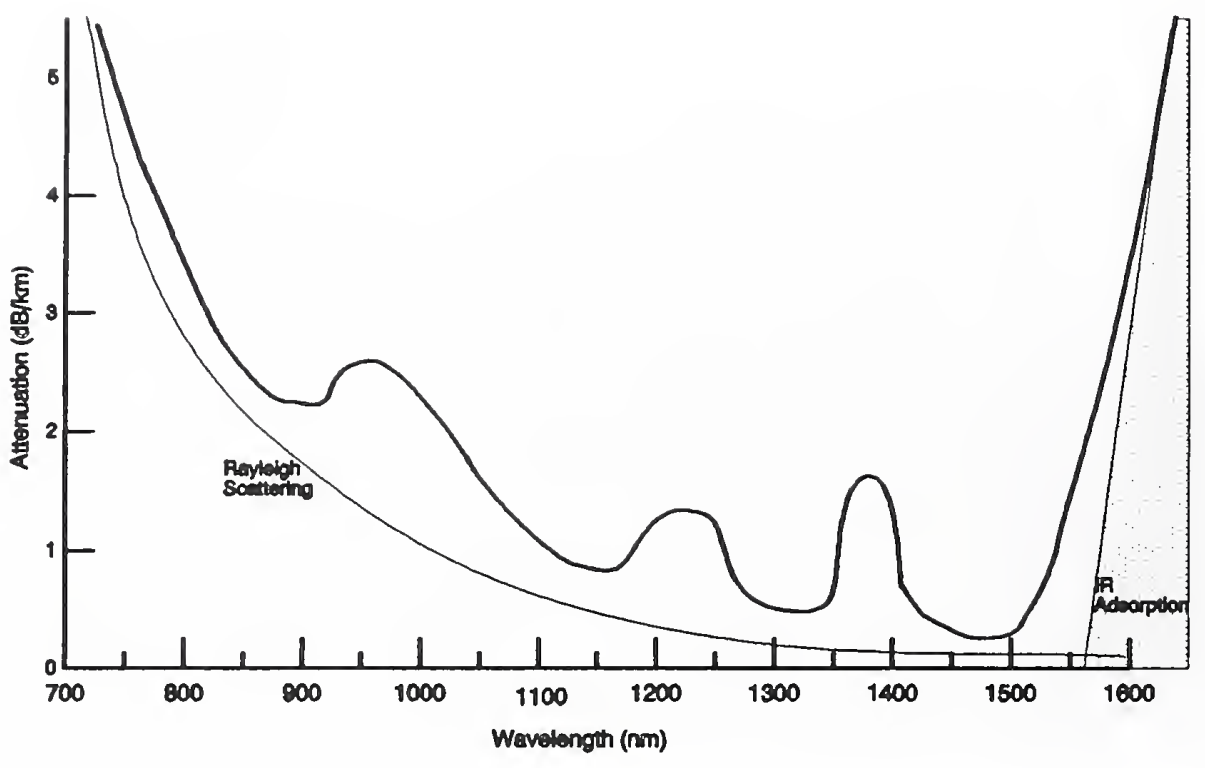

Figure 2 - Optical Fiber Attenuation vs. Wavelength

parison, the entire bandwidth now allocated by the International Telecommunications Union for radio transmission is $275 \mathrm{GHz}$ (much of which is, as a practical matter, of little communications use). The bandwidth allocated to the 68 VHF and UHF broadcast television channels by the FCC is $408 \mathrm{MHz}$. Thus current single mode fiber has an intrinsic bandwidth about 100 times greater than the entire radio spectrum and about 70,000 times greater than the television broadcast spectrum. With $64 \mathrm{kbit} / \mathrm{s}$ voice channels, the total busy period traffic of the current voice based US network is estimated to be $1,000 \mathrm{Gbit} / \mathrm{s}$ [71]. Therefore, even using inefficient modulation techniques requiring several $\mathrm{Hz}$ per bit, there is apparently more than adequate bandwidth in one fiber to carry the entire combined US voice load. There is, then, virtually unlimited bandwidth available in current commercial single mode fiber.

Commercially available transducers now limit the use of that bandwidth. Semiconductor laser diodes are the commonly used sources for long distance telecommunications. Since present commercial laser diodes resonate in several modes, they are not highly coherent. Optoelectronic switching is now limited to a few $\mathrm{Gb} / \mathrm{s}$ at best. Commercial digital transmission at about $600 \mathrm{Mbit} / \mathrm{s}$ is becoming routine and rates of several Gbit/s have been demonstrated in laboratories. Thus the best current commercial devices succeed in using only a tiny fraction of the inherent bandwidth of the current fiber. All current commercial fiber optic data transmission uses noncoherent on-off keying, but both heterodyne and homodyne data transmission have been demonstrated in many laboratories and offer the prospect of improved sensitivity and more efficient use of the spectrum of the fiber. It is safe to say that the present installed base of single mode fiber will be capable of carrying many times the present rates.

Other materials, primarily various heavy metal fluoride glasses, can shift the infrared absorption point to wavelengths as long as $3,100 \mathrm{~nm}$. This would allow losses perhaps as low as $.001 \mathrm{~dB} / \mathrm{km}$, which could very well make repeaterless transoceanic fiber links a reality [91]. They also could extend the low loss spectrum available for data transmission to nearly $100,000 \mathrm{GHz}$. The art of making $\mathrm{SiO}_{2}$ glass is so well developed, and its mechanical properties are so good, that new materials will have a very hard time displacing it, except in 
particular applications, such as transoceanic cables, where they offer a very great advantage. The only development likely to make present single mode fiber obsolete anytime soon is polarization preserving fiber. It is similar to conventional fiber, but has an asymmetrical core and only allows propagation of one polarization mode. Since optical mixers typically require similar polarization of the signal and the local oscillator, some coherent signalling schemes may require polarization preserving fiber. Even if coherent signalling requiring polarization preserving fiber becomes the preferred commercial technology, it is clear that the installed base of single mode fiber would still be a valuable asset, capable of carrying at least hundreds of $\mathrm{GHz}$ per fiber.

\section{Devices}

Although the fiber itself is marvelously good, the devices which are used with it are not so well perfected, and it is here that much progress can be expected.

\section{Sources}

Virtually all communications use of optical fiber relies upon some sort of semiconductor diode light source. These diodes are made of materials with suitable band gaps to generate photons of the desired wavelength (GaAs or AlGaAs in the $850 \mathrm{~nm}$ region, InGaAs or InGaAsP in the $1300 \mathrm{~nm}$ region, or InGaAsP in the $1550 \mathrm{~nm}$ region). These diodes may be lasers, but Light Emitting Diodes (LEDs) are frequently used as a source for multimode applications. LEDs have lower power densities than lasers which are nearly always used with single mode fibers. Laser Diodes (LDs) are the usual source for single mode links. In both LEDs and LDs photons are created by the recombination of charge carriers in the active layer of a forward biased diode. The laser, however requires a resonant cavity structure, so that recombinations stimulated by photons occur and the device lases. The cavity is formed by cleaving the surfaces of the semiconductor along parallel crystal planes.

Simple laser diodes may resonate in several different modes and their spectrum, consequently, is a series of closely spaced spikes. The spectrum, while far narrower than that of an LED, is still too broad (typically 1 to $5 \mathrm{~nm}$ linewidths) for coherent modulation, and is sufficiently broad that chromatic dispersion can be limiting in very long data links. LDs tend to be very temperature sensitive and both control of the bias current and thermoelectric cooling are often used to regulate LD temperatures.

As noted above, the multiple modes and undesirable broad linewidths of current commercial LDs limits their use in coherent systems and their performance in noncoherent long distance links. For this reason, considerable research is being devoted to narrowing the linewidths of laser sources, generally using optical feedback. External Cavity Lasers (ECL) have demonstrated $3 \mathrm{~dB}$ bandwidths down to several $\mathrm{kHz}$ [62]. Moreover such ECLs permit tuning of the frequency of the laser by tuning the cavity [114]. Single wavelength Distributed Feedback (DFB) lasers with a diffraction grating providing optical feedback at a fixed frequency are commercially available for long distance communications [22]. Two section DFB lasers, whose wavelength can be varied by injecting a small modulation current into one of the two sections, and distributed Bragg reflector lasers, in which at the frequency selectivity is provided by a diffraction grating etched into a waveguide monolithicially coupled to the light emitting region, are two types of tunable wavelength lasers which have been demonstrated [22].

In addition to marking the first demonstration of low loss fiber, 1970 saw the first demonstration of continuous room temperature operation of a semiconductor laser at Bell Labs [11]. Progress has not been quite as rapid in semiconductor lasers as in fiber and 
current commercial semiconductor LDs are hardly mature products. A detailed survey of all the developments in frequency and linewidth control for semiconductor LDs is beyond the scope of this paper. Such a variety of experimental systems has been demonstrated and so much research is being devoted to this, however, that it seems highly probable that commercially practical, very narrow linewidth, tunable lasers will be developed in the next few years.

\section{Amplifiers.}

Since optical links may have total bandwidths which exceed the capability of electronic components, direct optical amplifiers will be very useful. Without them, signals are now converted back to electrical form and then retransmitted to overcome transmission losses. If wavelength multiplexing is used to combine many signals, each near the electrical bandwidth limits, into one optical signal, repeating them in this way becomes very complex. Optical amplifiers are also required to overcome the losses of successive switching elements in optical switching systems. Laser amplifiers have been demonstrated in both semiconductor and glass fiber media.

Two kinds of Semiconductor Laser Amplifiers (SLA) have been demonstrated, travelingwave amplifiers (TWA's) and Fabry-Perot amplifiers (FPA's). FPA's have a large facet reflectivity forming a resonant cavity and therefore support several resonant modes and can only amplify signals at those frequencies. TWAs have much lower facet reflectivity, amplify in a single-pass and exhibit a wide gain bandwidth and have been shown to have superior characteristics to the FPA in both signal gain saturation and noise characteristics [81]. Semiconductor TWAs were demonstrated as early as 1982 [85], and broad-band, high-output-power, low noise and polarization insensitive signal-gain have been shown to be achievable in TWA's. As many as 5 laser TWAs have been cascaded to provide communications at $565 \mathrm{Mbit} / \mathrm{s}$ through a total loss of $112 \mathrm{~dB}$ with differential phase shift keying (DPSK) heterodyne detection [66]. A multiwavelength system has been demonstrated using a semiconductor TWA to provide between $6 \& 8 \mathrm{~dB}$ of gain for 20 equally spaced $1 \mathrm{Gbit} / \mathrm{s}$ channels spanning 1.527 to $1.561 \mathrm{~nm}$. Although not yet widely used in operational distribution systems, semiconductor TWAs operating at 1.3 or $1.55 \mathrm{~nm}$ are now commercially available [40].

Amplification over bands of $50 \mathrm{~nm}$ or more are achieved with SLAs. Therefore a single amplifier can amplify many densely spaced frequency division multiplexed channels. The principal noise phenomenon is SLAs is spontaneous emission noise, which can be held quite low. The noise characteristics of SLAs are so good, that it has been calculated that it is possible to build a $1 \mathrm{Gbit} / \mathrm{s}$ link $20,000 \mathrm{~km}$ long, without signal regeneration, if we can eliminate fiber dispersion (for example by using narrow linewidth sources near the zero dispersion wavelength of the fiber) [42].

Laser amplifiers can also be constructed in fiber using the Raman or Brillouin effects. In the Raman effect a molecule adsorbs a photon at one frequency and emits one at a shifted frequency. Raman effect broadband amplifiers can be constructed by appropriately doping fiber (often with erbium for operation in the $1500 \mathrm{~nm}$ region), then "pumping-up" the amplifier with a laser at some frequency well out of the signal range. Early experimenters used gas lasers as pumps, however fairly inexpensive AlGaAs semiconductor LDs operating at $807 \mathrm{~nm}$ have been used as a pump [111]; this is a three-level laser system, and comparatively inefficient [40]. Lasers diodes operating at $1480 \mathrm{~nm}$ have also recently been used to pump an $\mathrm{Er}^{3+}$ fiber amplifier with a $20 \mathrm{~dB}$ gain at $1560 \mathrm{~nm}$ [10]. Experiments have been conducted using Er-doped laser amplifiers in high bandwidth communications circuits [23]. 
In the Brillouin effect optically-induced polarization interacts with the acoustic vibrations of the glass producing light shifted by the acoustic frequency (about $10 \mathrm{GHz}$ ). Peak Brillouin gain in $\mathrm{SiO}_{2}$ is more than 2 orders of magnitude greater than Raman gain, but the frequency shift and gain bandwidth are much smaller. Because of the narrow bandwidth of the Brillouin effect, very narrow band (about $20 \mathrm{MHz}$ ) amplifiers are possible with single frequency pumps, while dithering of the pumps or doping the fiber with Ge can produce bandwidths in the hundreds of $\mathrm{MHz}$. Rather elegant tunable direct detection receivers have been made which allow a $128 \times 128$ channel network with channel spacings as small as $1.5 \mathrm{GHz}$ at a data rate of $150 \mathrm{Mbit} / \mathrm{s}$, using a Brillouin amplifier both as a tunable filter and an amplifier [94]. Equally elegant homodyne detection using Brillouin amplifiers as both filter and amplifier have also been described [6], [84].

Thus broadband laser traveling-wave amplifiers, broadband Raman fiber amplifiers, and tunable, narrowband Brillouin amplifiers have all been demonstrated, providing an array of choices to future designers. The initial commercial use will probably be to extend power limited point-to-point links. As with any amplifier, the noise introduced by the amplifiers (primarily caused by spontaneous emissions) as well as the dispersion of the fibers will limit the number of cascaded stages.

\section{Detectors}

A variety of photodetectors has been developed, including vacuum tube photomultipliers and photoresistors. Today, and for the foreseeable future, the bulk of communications circuits will use either semiconductor PIN (p-type, intrinsic, n-type) photodiodes or avalanche photodiodes. The PIN diode has a comparatively wide undoped intrinsic layer with no free charges sandwiched between $p$ and $n$ regions. Since the intrinsic layer is wide and has no charge carrier, it has a high resistance and a reverse bias creates a strong electric field across it. Because of its width, most incident photons are adsorbed by the intrinsic layer and photons with sufficient energy to raise electrons across the bandgap of the material create an electron-hole pair, allowing a current to be carried. Si has a bandgap of $1.1 \mathrm{eV}$, which is practical in the $850 \mathrm{~nm}$ region, but too large for the 1300 and $1550 \mathrm{~nm}$ regions, which require $\mathrm{Ge}$ or $\mathrm{InGaAs}$ detectors. At suitable wavelengths, the current across the reversed biased PIN diode consists of a dark current, of thermal origin, present when there is no incident light, plus a current proportional to the incident photon flux.

Avalanche photodiodes (APD) are usually similar in general structure to PIN diodes and are made of similar materials. APDs, however are designed to operate at high reverse biases, as much as several hundred volts. Because of the stronger electric field the free holes and electrons are accelerated to the point where they gain enough kinetic energy to create other electron-hole pairs when they collide with neutral atoms. This avalanche effect provides internal amplification, while adding less noise than would be possible with external amplifiers, decreasing the relative significance of thermal noise and increasing the sensitivity of the detector.

Two distinct types of noise are present in both types of detectors. Thermal (or Johnson) noise is caused by the random thermal motion of atoms and electrons which can cause a net flow of charge to move toward the electrodes at any instant, creating a randomly varying current which becomes significant at very low received signal levels. Thermal noise varies directly with absolute temperature, and can be reduced, in principle, by cooling detectors. Shot (or quantum) noise results from the discrete nature of photons and electrons and therefore increases directly with the signal power. It is shot noise which fundamentally limits receiver sensitivity. 
At high signal power levels, the random noise contribution is reduced to insignificance by shot noise, and the detector is said to be shot-noise or quantum limited. In this region, the signal-to-noise ratio (SNR) varies linearly with the received signal power and the signal is strong. When power levels are sufficiently low, thermal noise may dominate and the detector is thermal-noise-limited. In this region, the SNR varies as the square of the received signal power, therefore small changes in signal power can be significant. Both thermal and shot noise have a broad uniform spectrum, so it is important that receivers limit their bandwidth to little more than that of the signal, to limit the noise.

Shot noise limited operation provides superior reception, however with APDs, shot noise increases as gain is increased. Therefore, at any input power level an optimum level of gain exists for APDs, which maximizes the SNR. The gain of APDs is easily controlled by changing the reverse bias voltage.

\section{Switches}

While early fiber switches have often been done with mirrors, electro-optic switching is possible. The material most commonly used is $\mathrm{LiNbO}_{3}$, [43] which has a strong electro optic effect, that is its index of refraction changes in an electric field. Two closely spaced waveguides are formed by the diffusion of $\mathrm{Ti}$ in a $\mathrm{LiNbO}_{3}$ substrate, in a fashion similar to a unidirectional coupler. The channel length is adjusted so that (ideally) all light is coupled from one channel to the other when no electric field is applied. When a suitable electric field is applied the refractive indices of the two channels change and no light is coupled from one waveguide to the other. Such a device is illustrated in figure 3.

One disadvantage to such switches is that they operate in only one of two states, "bar," where the each input port is connected to the corresponding output port and "cross", where the outputs are reversed, but have no "broadcast" state, in which one of the inputs is connected to both outputs. Another limitation is that such devices exhibit undesirable high loss and crosstalk properties, and the largest arrays which have been demonstrated so far
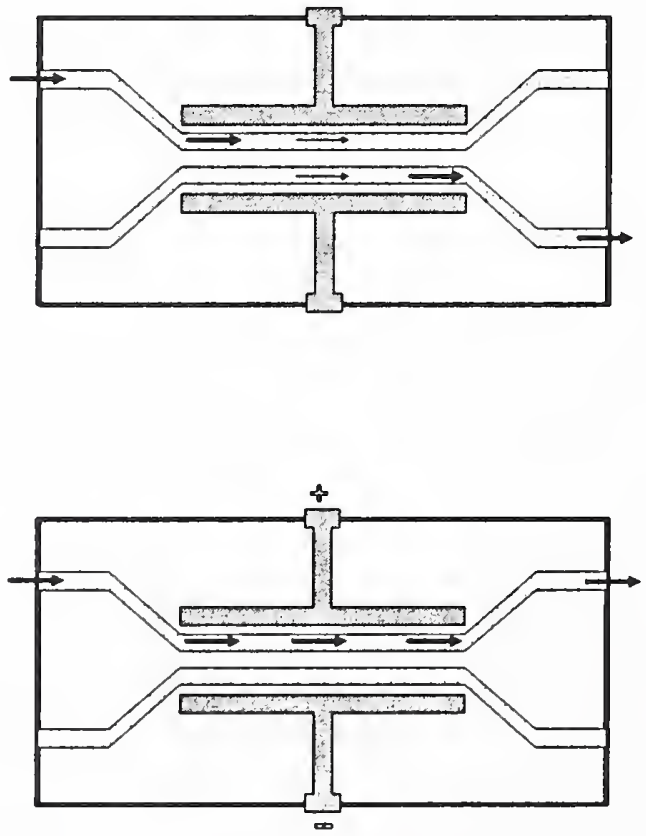

Figure 3 - Lithium Niobate Switch 
are $8 \times 8$ arrays [25]. Another disadvantage is the need to maintain polarization to limit switching voltages. While commercially available products have so far been $\mathrm{LiNbO}_{3}$ devices, polymers have also been developed which demonstrate an electro-optic effect.

Another way to make an electrically controlled photonic circuit switch is to combine a passive optical coupler with an array of semiconductor laser amplifiers. By electrically activating the amplifiers an input could be selectively routed to one or more outputs. Theoretical analysis suggests that crosstalk can be held to low values (less than $-60 \mathrm{~dB}$ ) and large arrays of cascaded devices are possible even with fairly low gain amplifiers [25].

While most work to date in integrated optical switches has been done with $\mathrm{LiNbO}_{3}$, switching devices and modulators made with III-V $\mathrm{V}_{1}$ semiconductors would be particularly attractive, because they could be monolithically integrated with active devices such as laser diodes and photodiodes. If large scale integration of such devices could be perfected, then the structures of contemporary computers and switches could be replicated photonically. However this technology is in its infancy and, "at present, it is difficult for engineers involved in telecommunications research and development to imagine a realistic photonic exchange system, because they have too much knowledge about its complexity and the development speed of microelectronics...implementation of the photonic exchange system is very far in the future," [52].

\section{Modulators}

While direct modulation of lasers is desirable for simplicity sake, rapid modulation may cause a variety of effects, particularly "chirping" as the laser switches. Fast external $\mathrm{LiNbO}_{3}$ switches have been widely used as external modulators in high bandwidth experiments [59], [109], [96]. External $\mathrm{LiNbO}_{3}$ modulators should be capable of supporting data rates between 1 to $10 \mathrm{Gbit} / \mathrm{s}$, while for direct modulation of lasers the voltage required from a fixed impedance source to maintain a constant modulation depth increases quadratically with frequency [59].

\section{Filters}

While there are many ways to filter light, including gratings [58], prisms, and the absorption properties of materials, one stands out as particularly important, the Fabry-Perot interferometer, sometimes called an "etalon" which can readily be made tunable. The FabryPerot filter is simply an optical waveguide cavity with partially silvered mirrors at the ends. The transmission function is periodic, with transmission peaks spaced by the Free Spectral Range (FSR), which varies inversely with the length of the cavity. The cavity length can be made variable and controlled with the piezoelectric effect, or even a voice coil, and a transmission peak can be scanned through one FSR by tuning through one half-wavelength. The ratio of the $-3 \mathrm{~dB}$ passband width to the FSR, is called the "finesse" (F) and is analogous to the $\mathrm{Q}$ of an electrical filter.

If frequency shift keying (FSK) modulation is used, the Fabry-Perot filter can also serve as a demodulator. Fabry-Perot filters with an $F=200$ and a $5 \mathrm{~dB}$ insertion loss have been

1 The "III-V" refers to the III and V groups of elements in the periodic table. The semiconductor materials with band gap energies corresponding to 800 to $1600 \mathrm{~nm}$ photons are alloys of group III elements, such as Al, Ga or In and As, a group V element. 
demonstrated, which would permit a 33-channel FSK frequency division multiplexing network [56]. Fabry-Perot filters can be cascaded to increase F and a system of two cascaded FP filters has been demonstrated with an $F$ of 675 , which is sufficient to tune and demodulate about 225 frequency shift keying channels [57].

Other kinds of tunable filters also have been used, including Mach-Zehnder interferometers. Acoustooptic tunable filters have been used to tune 9 distributed feedback lasers with $4.5 \mathrm{~nm}$ spacings, in the 1500 to $1600 \mathrm{~nm}$ region [15]. Narrow-band Brillouin fiber amplifiers also make a very attractive, tunable, narrow band filter, as well as an amplifier, as noted above.

\section{Coherent Detection}

Present commercial fiber optic links use non-coherent direct detection of signals. They are roughly analogous to the spark gap transmitters and detectors used by Marconi and other early radio experimenters, and are similar in principle to the signalling used in "baseband" electrical networks. Modern broadcast radio transmission and "broadband" electrical networks, however, employ coherent detection, which have the following significant advantages:

- They allow shot noise limited operation at lower signal levels, improving the sensitivity of receivers by about $10 \mathrm{~dB}$.

- After the detector, all signal processing takes place in a single fixed intermediate frequency band, simplifying the design of the demodulator and any IF amplifiers.

- The filtering inherent in the IF operation tends to remove broad spectrum background noise present with the signal. This is less significant in optical communications than in radio communications, since there are less sources of such noise.

- Frequency division multiplexing (FDM) is simplified because it generally proves to be easier to make a widely tunable local oscillator, followed by a fixed frequency narrowband filter, than a narrowband tunable filter.

Thus there are two motivations for interest in coherent detection of optical signals: to increase optical power budgets (thereby allowing either more stations to be attached to one broadcast transmitter or increasing distances between repeaters) and to increase the capacity of optical circuits and networks by the frequency division multiplexing of many signals on one fiber. The increase of capacity by FDM is probably the stronger reason. Optical amplifiers allow far greater increases to link power budgets than the $10 \mathrm{~dB}$ or so advantage of coherent over noncoherent detection.

The receiver commonly used for coherent detection is the heterodyne receiver illustrated in figure 4. In this receiver a remote, modulated, information bearing signal is combined with a polarization aligned signal from a variable frequency local oscillator, using grating or a partially silvered mirror. This combined lightwave signal is used to illuminate the junction of a photodiode, and the power of the resulting electrical signal is proportional to the square of the light signal. By adjusting the frequency of the local oscillator it is possible to match the frequency of the difference term to the passband of a conventional electrical microwave filter and do all further processing of the signal electrically in a single fixed frequency band. Thus a coherently modulated optical signal is converted to an intermediate frequency coherently modulated electrical signal and demodulated. 


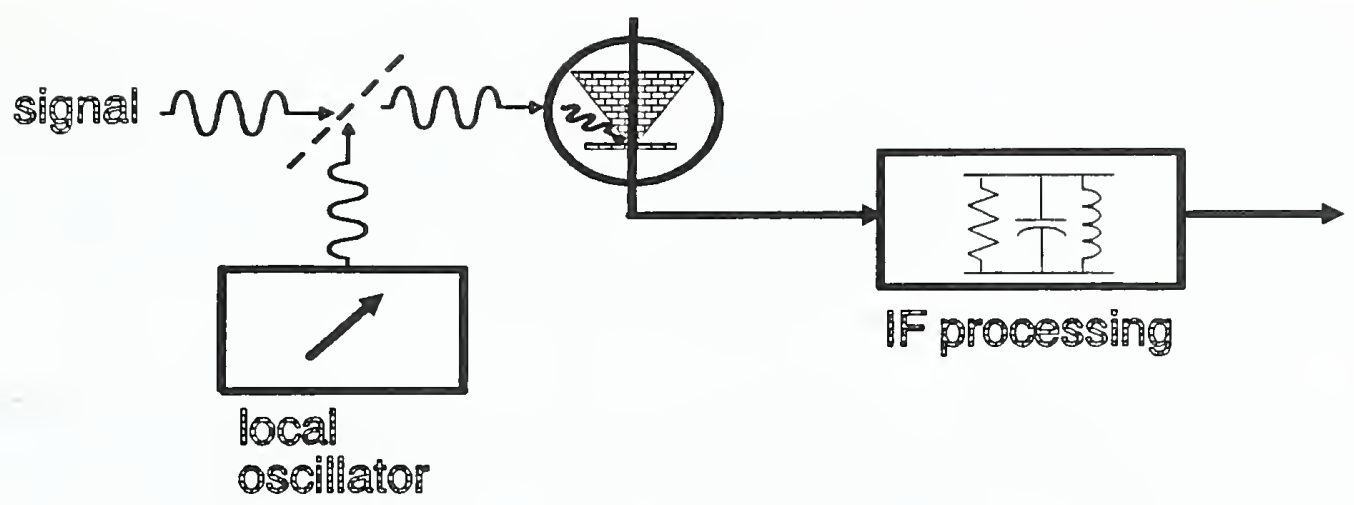

Figure 4 - Heterodyne Receiver

It is possible to match the local oscillator to the carrier frequency, directly creating a baseband signal coming from the photodetector; such receivers are called homodyne receivers and offer a theoretical $3 \mathrm{~dB}$ power advantage over heterodyne receivers. The homodyne receiver, however, requires an optical phase lock loop to lock the local oscillator to the signal phase, which is difficult to achieve. The heterodyne receiver is therefore generally easier to realize, is used for most electrical receivers, and will most likely be the commonly used optical receiver as well.

If the information signal is $A_{s i g} \cos \omega_{1} t$ and the local oscillator signal is ALO $\cos \omega_{2}$ t, then the intermediate frequency (IF) difference signal is [62]:

$$
2 \mathrm{~A}_{\text {sig }} \mathrm{A}_{\text {LO }} \cos \left(\omega_{1}-\omega_{2}\right) \mathrm{t}
$$

Since the IF signal is proportional to the product of the input signal and the local oscillator signal, and the local oscillator signal can be made quite large, the IF signal can be made as high as necessary to make thermal noise in the detector and subsequent components insignificant, and the limiting noise becomes the shot noise in the detected photo-current. While direct detection can, in principle, be made as efficient as coherent detection by eliminating thermal noise (say by cooling the detector to near absolute zero), as a practical matter, in the 1300 and $1500 \mathrm{~nm}$ regions the best direct detectors are now limited by thermal noise to sensitivities at least 40 times the theoretical shot noise limit of 10 photons/bit at a bit error rate (BER) of $10^{-9}$, while coherent detection has come within a factor of 2 of the theoretical limit [62] at the same BER.

While most heterodyne transmitters and receivers implemented to date have used separate transmitter and receiver local oscillator lasers, it is possible to use a single semiconductor laser as the local oscillator, mixer, detector and transmitter. Interference between the lasing mode and the injected received signal modulate the rate of stimulated carrier recombination and thereby produce a change in the injection current, from which the received signal can be recovered. The performance demonstrated to date with such a receiver/transmitter has not been nearly as good as with more conventional approaches, largely because of impedance mismatches between the laser junction and the low-noise first amplifier. However, it is an intriguing approach to implementing a "self-heterodyne" receiver/transmitter and full duplex operation over a single fiber has recently been demonstrated at 40 Mbit/s [64]. 
At least three basic ways exist to modulate a carrier coherently: phase shift keying (PSK), frequency shift keying (FSK) and amplitude shift keying (ASK), and several variations on these are possible. References [62] and [88] summarize the various theoretical shot noise limited sensitivities for most of the alternatives, and summarize the results of many coherent transmission experiments. With differential PSK, a sensitivity of $-56.3 \mathrm{dBm}$ has been demonstrated for a BER of $10^{-9}$ and a rate of $400 \mathrm{Mbit} / \mathrm{s}$, allowing transmission through a distance of $260 \mathrm{~km}$. At $2 \mathrm{Gbit} / \mathrm{s}$, and similar modulation methods and error rates, a sensitivity of $-39 \mathrm{dBm}$ has been demonstrated allowing a distance of $170 \mathrm{~km}$.

While a great deal has been demonstrated in the laboratory, and coherent transmission is the subject of intense current research, there are significant obstacles to commercial exploitation of coherent systems. Highly coherent, very narrow line width sources are required, and while there are several approaches which permit this, including distributed feedback (DFB) lasers, external cavity (ECL) lasers and distributed Bragg reflector (DBR) lasers, these remain, for the moment, laboratory curiosities, not yet commercially available. When used as local oscillators or as sources in tunable wavelength division multiplexing networks, the frequency of such devices must also be precisely adjustable. Since precise control of frequencies, and elimination of multiple modes require complex structures and precise control of dimensions, temperatures of sources must generally be precisely controlled. Since these are quite high power density devices with complex structures, bringing practical devices to high volume commercial production is challenging.

Another difficulty is that optical mixers require that the received signal and the local oscillator have the same polarization. Ordinary single mode fiber changes the polarization of the output, and this change varies over time. Approaches to handling polarization changes include tracking the polarization of the received signal as it changes, using polarization preserving fiber (generally made with an elliptical core), and polarization diversity detectors, which use two detectors and pay a $3 \mathrm{~dB}$ theoretical penalty for being insensitive to polarization [88]. Tracking polarization changes adds complexity, while there is a good deal of cylindrically symmetrical fiber already installed and it will complicate connectors greatly if elliptical cores must be rotated into alignment at each connector or field splice.

In broadcast networks with many distant transmitters, the frequency drift of the transmitters is a serious problem, because they must each generate the same stable, densely spaced "comb" of frequencies. Laser frequencies are sensitive to both temperature and injection current changes, even in single mode lasers. Frequencies change by $10-20 \mathrm{GHz} / \mathrm{degree} \mathrm{C}$ and by 1-5 GHz/mA [54]. Solutions demonstrated to this so far include using the resonances of a Fabry-Perot interferometer or the absorption spectral lines of gaseous atoms or molecules as a reference to which the laser is locked $[73,87,50]$.

Coherency will probably first be used commercially to implement very long distance, or very high bandwidth FDM, point-to-point links. Applications such as underwater cables, where the cost of running fibers is high, are particularly favorable, because of their relative insensitivity to the initially high cost of coherent components. As the cost of coherent detection falls, however, it will be used in applications which are more sensitive to the cost of components, such as local networks. The obstacles to commercial use of coherent fiber optics are formidable. However, significant commercial exploitation of coherent technology within a decade or two does not appear to require progress at a rate greater than has been achieved over the past two decades in optical fiber and components. 


\section{Conclusion}

It seems clear that optical devices which duplicate the functionality of nearly all analog electrical devices have been demonstrated and will be developed commercially. Entirely optical digital logic is also possible, but its development is still in earliest infancy. While it is difficult to predict the exact pace at which these components will be developed and introduced commercially, much research is under way.

While photonic switching devices which replicate the complexity of their electrical equivalents appear remote, the components required for coherent detection and efficient frequency division multiplexing are likely to be commercially available soon. The sheer volume of research on coherent communications and optical amplifiers is impressive: from the January 5 to the June 8, 1989 issues of Electronics Letters, nine separate coherent transmission experiments were reported at rates from $140 \mathrm{Mbit} / \mathrm{s}$ to $5 \mathrm{Gbit} / \mathrm{s}$, three wavelength or frequency division multiplexing experiments were reported, along with 8 transmission experiments using inline semiconductor laser amplifiers and 2 using inline fiber amplifiers. While there are apparently no data links in commercial operation using either optical amplifiers, wavelength division multiplexing or coherent detection, with this level of effort, commercial application cannot be far off. 


\section{Present LAN Architectures}

Many present topologies and architectures seem unsuited to future very high bandwidth networks. Current computer LAN topologies, illustrated in figure 5, include:

- rings

- dual rings

- dual rings of trees

- transmissive stars

- dual buses

- linear buses

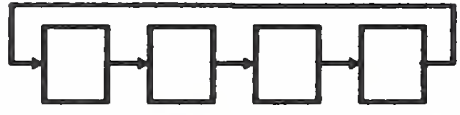

(a) Ring

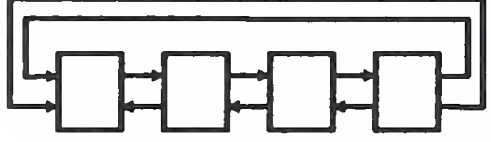

(b) Dual Ring

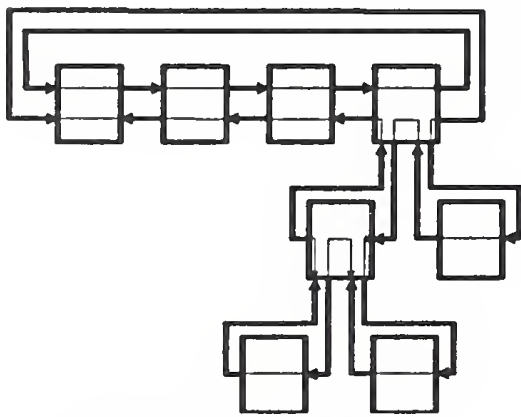

(c) Dual Ring of Trees

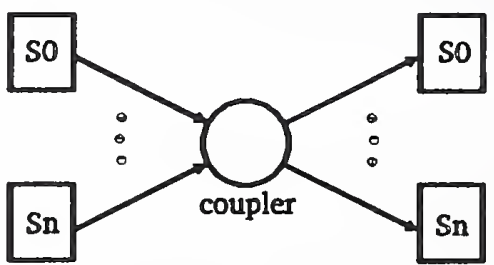

(d) Transmissive Star

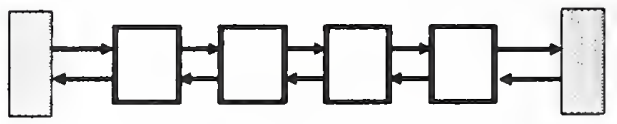

(e) Dual Bus

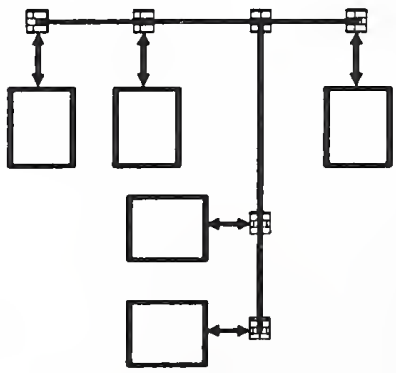

(f) Linear Bus

Figure 5 - Common LAN Topologies 
These topologies are employed with a medium access mechanism (MAC) which imposes some sort of time division multiplexing, to share the medium, usually at a frame or packet level. For example, within the ring topology we find three distinct medium access and time division multiplexing schemes:

- token passing rings

- slotted rings

- shift register insertion rings

In token passing rings, which include the popular IEEE 802.5 and FDDI standards [27], a special packet, called a token, is passed around the ring. A station wishing to send waits for the token, "captures" it, then sends his packet(s) and finally releases the token.

In slotted rings the transmission path around the ring is made an integral number of fixed size packets or "slots" long. A station seeing a slot marked empty marks it full and fills it with data. The best known of the slotted rings is the "Cambridge Ring" [46] which was developed at Cambridge University and has been commercialized in the UK. FDDI-II inserts a slotted ring, with $125 \mu$ s slot times underneath a logical token ring, to provide both an isochronous circuit switched and a token packet service.

In a shift register insertion ring each station contains a shift register buffer as long as the longest packet it can send. When the station wishes to send, it looks for an idle space between packets then begins transmitting. If a packet is received from upstream while the station is transmitting, it is shunted into the shift register and then repeated after the station is done transmitting its own packet. While experimental shift register insertion rings have been built [47], none have apparently been commercialized.

All three of these networks are physically connected as rings and transmit data in ring fashion, using different MAC disciplines to achieve time division multiplexing. In the case of FDDI and IEEE 802.5, the unit of multiplexing is a variable size packet. In FDDI-II, however, it is possible to multiplex circuits of as fine a granularity as $8 \mathrm{kbit} / \mathrm{s}$, on a 100 Mbit/s carrier, by assigning 1 bit from each slot. All three suffer from the same fatal flaw which limits their utility in implementing a very high bandwidth network: a great deal of very fast logic is required in each station to run at the full aggregate bandwidth of the network. Even at the comparatively low FDDI rate of $100 \mathrm{Mbit} / \mathrm{s}$, the development of protocol chips has proved challenging. It appears that a higher rate for FDDI of from 400 to $1000 \mathrm{Mbit} / \mathrm{s}$, will be practical in a few years, probably using some GaAs front end logic. Beyond that it is hard to envision radical growth.

Another network architecture, proposed for use in Metropolitan Area Networks, is the Dual Bus Dual Queue proposed standard of IEEE 802.6 [116]. DQDB uses the 48-byte (plus 5-bytes of header) Asynchronous Transfer Mode (ATM) cell planned for B-ISDN, so it is interesting, as the first precursor of B-ISDN and as an example of a distributed B-ISDN packet switch. Its performance is less degraded by long distances than is FDDI [107], [68], but it still requires every station to examine every packet on the network at the aggregate rate of the network.

Baseband bus and star broadcast networks, rooted tree networks and dual bus networks, all suffer from more or less the same problem: a significant amount of digital logic must work at the aggregate rate of the network. Since the bandwidth of the fiber would support much higher rates than semiconductor logic can support, this means that the speed of the semi- 
conductor logic is limiting. This problem is inherent in any time division multiplexing protocol, where the logic elements are orders of magnitude slower than the medium bandwidth. Many Gbit/s networks using rings or dual buses will not be practical at least until large scale photonic integrated logic can be built. This appears quite distant,.

Many present LAN architectures, then, are not well suited to fully exploiting the bandwidth potential of optical fiber. There are, however, a few "broadband" LANS, using star or bus topologies, which use electrical frequency division multiplexing (FDM) to provide a number of separate channels on one cable. Within each channel they often use a MAC protocol similar to one of the baseband protocols, such as token passing, or Carrier Sense Multiple Access (CSMA). These, although not particularly widely used, point the way to one means of more fully exploiting the fiber optic bandwidth with individual stations which are much slower than the fiber: optical FDM 


\section{Centralized Switches}

Several taxonomies have been proposed for classifying switches. One, focusing on photonic switches, proposes classifying them according to the physical switching principles used (optical or optoelectronic), the multiplexing system used (space division, modulation division or carrier division) or the optical configuration of the switch system (centrally switched, optically extended or distributed) [65]. Another taxonomy classifies switches by whether they use dedicated or shared links, by whether data units are transmitted on an assignment based or statistical manner, by whether routing control is centralized or distributed, by whether switching elements are buffered or unbuffered, or by whether space switching, time switching, frequency switching or address filtration is used as the switching technique [19].

This section will focus on centralized switches, that is switches located at internal switching nodes within the network, rather than switching which involves switching decisions primarily at the endpoint stations. We consider the likely alternatives for such switches to be classified as either circuit switches or packet switches and the physical switching possibilities to be either entirely electronic switches, electrically controlled photonic switches, or entirely photonic switches.

In entirely electrical switches, incoming optical signals are converted to electrical signals before switching. Multiplexed optical signals may be demultiplexed optically before conversion or electrically after conversion. In electrically controlled optical switches, the control of the switch and routing are electrical, but the actual switching is done with photons in the optical domain. In entirely photonic switches all logic, switching and control is done photonically. The consensus of researchers is that complex, entirely photonic switches will not be realizable within the next two decades $[71,52,43]$ and they will therefore not be considered further.

Traditional telephone central office switches have been circuit switches. The earliest were electromechanical switches. Eventually, by the 1960's, these were supplanted by a generation of computer controlled electromechanical switches, and this was followed in the 1970's by computer controlled, digital semiconductor switches, which dominate the current switching plant. The latest of these switches strive for compatibility with the ISDN standards. They offer the prospect of all digital end to end transmission of $64 \mathrm{kbit} / \mathrm{s}$ basic rate voice or data circuits and either 1.544 (in North America) or $2.048 \mathrm{Mbit} / \mathrm{s}$ (in Europe) "primary" rate circuits, while preserving compatibility with the earlier generations of analog and preISDN digital equipment.

In the mean time, the computer industry, faced with high costs for low data rate point to point circuits, developed packet switching. It found two manifestations. The first was store and forward packet switches which were developed using general purpose computers as switches. The switches received packets from one low speed (typically 9.6 or $19.2 \mathrm{kbit} / \mathrm{s}$ ) long distance point to point circuit, buffered the packet, checked it and routed it on, over some other point-to-point link, to its eventual destination. This was done almost entirely in software, which was practical because the instruction rate of the computers used as switches was high compared with the speed of the data communications lines. This is the classic situation for packet switches: fast switches and slow communications links. Computational and switching resources are used to maximize the use of restricted communications bandwidth. 
The second manifestation of packet switching was Local Area Networks (LANs). They were developed to perform distributed packet switching, at much higher communication rates. In LANs packets are usually broadcast to all stations on the network, and the destination stations recognize those packets addressed to them. Two topologies and access mechanisms predominated, the Carrier Sense Multiple Access with Collision Detection (CSMA/CD) broadcast bus [67], and the token ring [13]. Much higher speeds (4 to 100 $\mathrm{Mbit} / \mathrm{s}$ ) were achieved because of the shorter distances and because the basic routing, access mechanism, error detection and address filtering operations were all done in dedicated hardware.

These LANs are distributed, time division multiplexed packet switches. Both have been adapted to optical fiber media. Distributed packet switching is now used as a means to share a fairly abundant, but still limited, communications resource. Although the CSMA/CD bus and the token ring are the most effective high data rate packet switches now in wide use, both approaches are ultimately limited to rates a tiny fraction of the fiber bandwidth, because the electrical circuits in each node must process the entire total data load of the network.

As a result of the development of powerful microprocessors, a great interest arose in building very powerful multiprocessor computers from large arrays of microprocessors. Packet switches suitable for connecting parallel computer systems were developed in the late 1970s. These ideas have subsequently been adapted to packet switches for long distance communications and Broadband ISDN (B-ISDN) is based on the high speed switching of short (48-byte) packets1, called Asynchronous Transmission Mode (ATM) cells [14, 37, 105, 33, 79]. The ATM cell is intended to be used for both isochronous services, which mimic circuit switching, and packet data switching on one carrier and switching fabric. Ideally voice, data and video services would be switched and distributed on one homogeneous network worldwide.

The construction of suitable very high speed packet switches, is however, a major challenge. Most approaches assume entirely electronic switching and attempt to use structures which introduce parallelism to meet the total data rate requirements of the switch.

\section{Banyan Switches}

The banyan (sometimes called "baseline") network, illustrated in figure 6, is the most common example of a class of topological equivalent multistage networks [113], which has been extensively used in multiprocessor applications and is commonly proposed for use in high speed packet networks [3]. It switches any of $2^{\mathrm{n}}$ inputs to any of $2^{\mathrm{n}}$ outputs, using $\mathrm{n}$ stages of 2-input x 2-output switches. The number of 2-input by 2-output switching elements is nlog2n. The switching elements need only be capable of 2 states, direct connection and crossed connection.

1 It may be premature to claim that it is settled that all B-ISDN will be based on the ATM. There has also been support for a Synchronous Transfer Mode (STM) service, and some European papers and B-ISDN trials seem to view this as the primary $\mathrm{B}-\mathrm{ISDN}$ service. The decision to pick a 48-byte cell is very recent (spring 1989), and the small cell size reflects concern about managing delay when using ATM cells to simulate circuit switched isochronous services. 


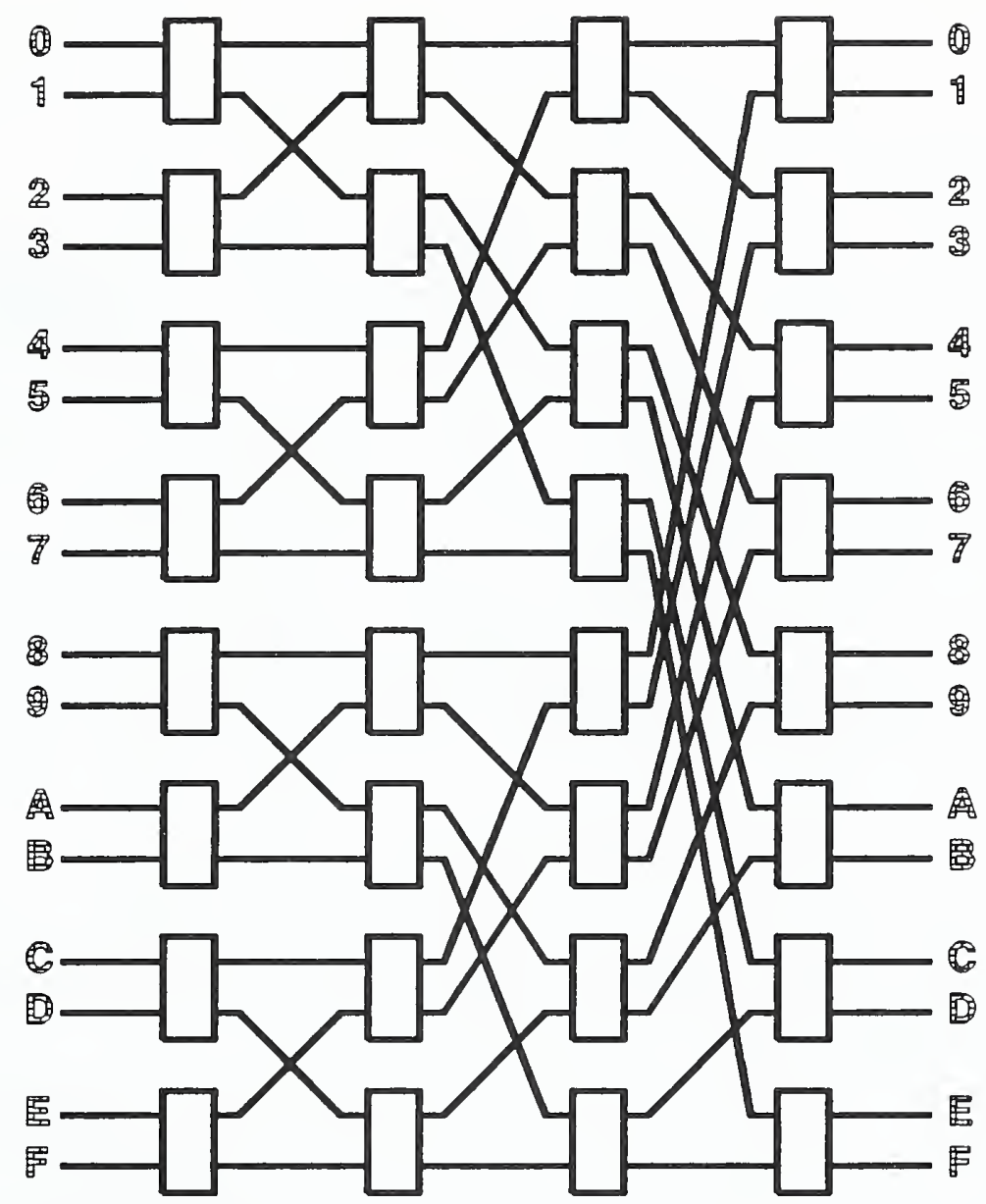

Figure 6 - 16 by 16 Banyan Switch

Banyan networks may be used for self routing packet switches as well as circuit switching. At each stage of the switch it is only necessary to examine one bit of the destination address to select the output port. To see this in figure 6, position one bit of the +binary destination address over each column of switches, from left to right. A " 1 " selects the lower switch output, and a "0" selects the upper. Start at any input port and follow the selected path.

There is precisely one path between any input and any output in a banyan switch. This has the desirable property that out-of-sequence packets between the same two terminals will not occur in a banyan packet switch. However, contention for intermediate links may cause connections between separate endpoints to interfere with each other, causing congestion in the switch. For example, in the 16 port banyan illustrated in figure 6 , the 1 to 8 path blocks the paths from 0 to 8 through $F$, the paths from 2 and 3 to 8 through $B$ and the path from 4,56 , or 7 to 8 and 9 . With a constant per port load, as $n$ increases the probability of congestion also increases. Simulation has shown that, when a buffer is inserted in each switching element to hold data in the event of conflict, packet banyan networks work well when traffic is uniformly distributed, but much less well for nonuniform traffic [3].

A switch is said to be "nonblocking" if packets or connections to different output ports do not block each other, and considerable effort has been expended to find ways to incorporate the banyan switching fabric into nonblocking switches, or in reducing the probability of blocking. No buffering for congestion is required in the nodes of a nonblocking network, 


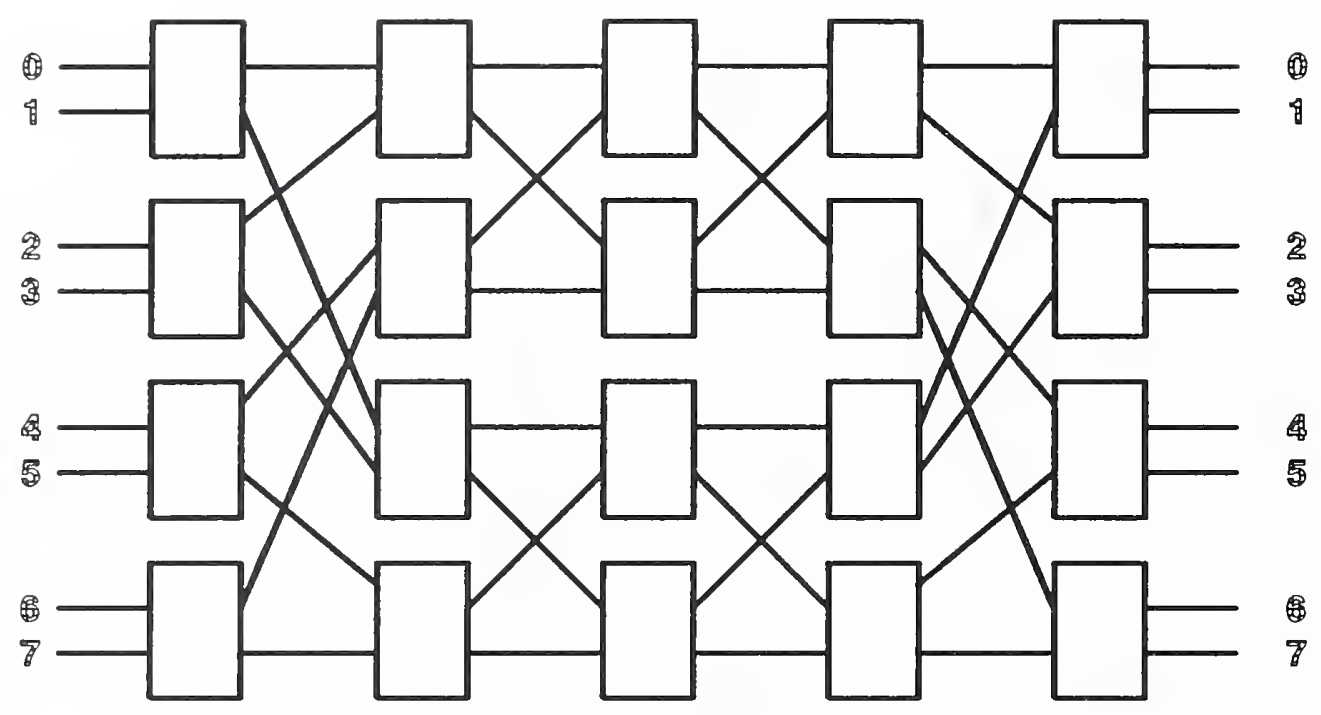

Figure 7 - The Nonblocking Benes Switch

unless there are output port conflicts; the nodes in a blocking network must contain buffering or collisions within the switch will cause packet loss.

An approach to improving the performance of such networks is to precede the switching network with a routing network, often identical to the switching network [3], [99], [101]. This cascaded banyan, sometimes called a Benes network, is illustrated in figure 7 for an $8 x$ 8 interconnection network. There are now $2^{\mathrm{n}}$ paths for every terminal pair. Typically, an external connection control processor assigns the routing for each connection at call setup, and all packets for that connection then follow that route. All packets in a call follow the same path maintaining packet sequence integrity and minimizing packet delay variation. Several algorithms are possible for assigning connection routes at call set up time, the simplest of which is random selection. By using state information to make selections, performance can be improved, and the network made completely nonblocking.

Another approach is to introduce one feedback loop for each input/output terminal [102]. Packets can be routed through the network once for distribution then fed back and switched, effectively replicating the cascaded banyan. A connection processor establishes a fixed route through the switching network at call setup. If possible, the route exits directly at the destination after one pass.

Performance of the banyan can also be improved by preceding the switching network with a presorting stage before the routing network [48], [49], usually a Batcher network [9], as illustrated in figure 8. By arranging the incoming packets with distinct addresses in ascending or descending order, the banyan network becomes nonblocking. When there is an output conflict, the second packet is delayed to the next cycle [49] or, in the STARLITE switch, one of the earliest such switches described, fed back into the beginning of the input queue [48], which has the disadvantage of possibly reordering packets. 


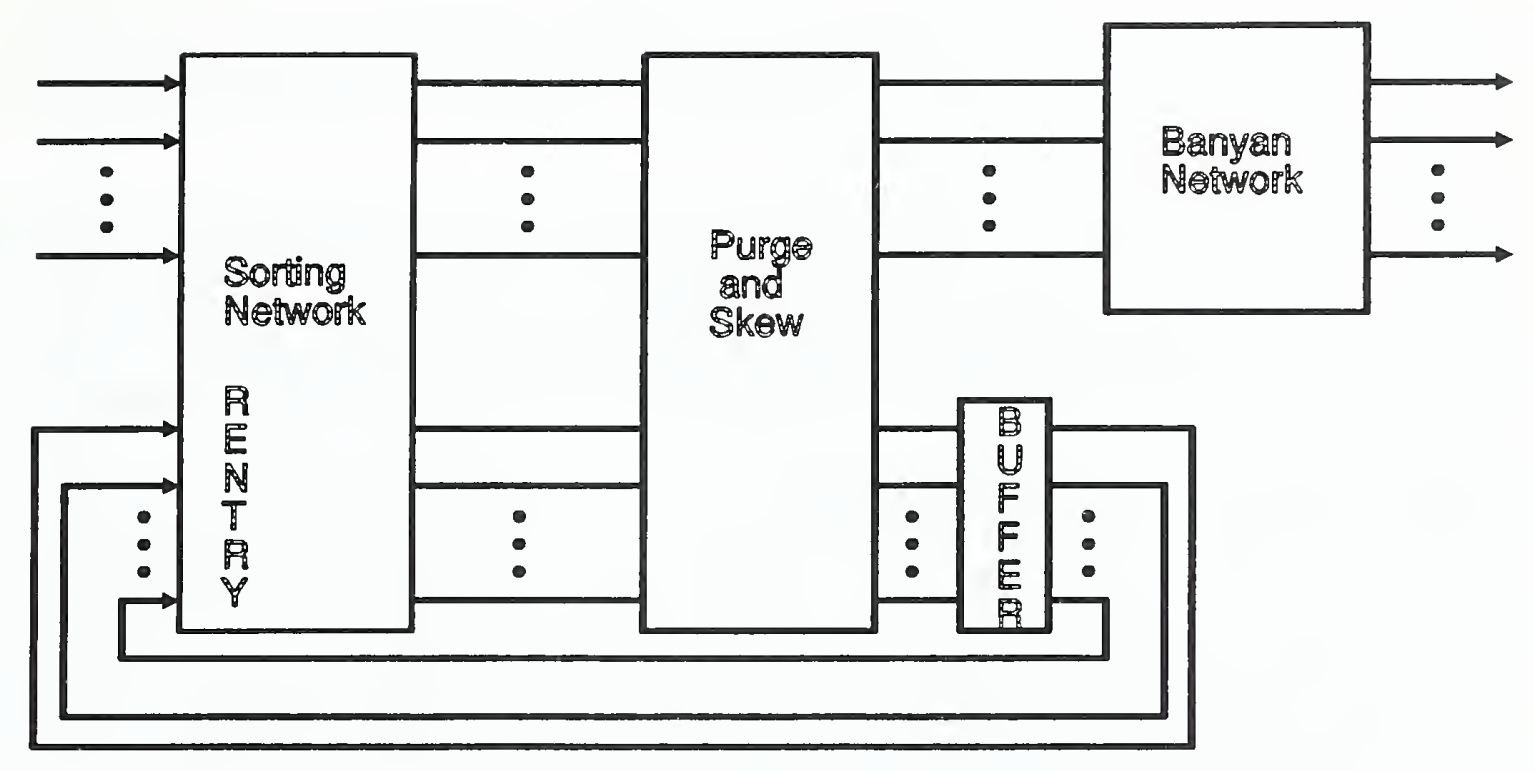

Figure 8 - The STARLITE Switch

To accomplish distribution of video to residences by high speed packet switches, a broadcast capability must be added to the switch, so that one input signal can be distributed to many outputs. Turner [100] proposes a large switch be made of an array of the switch fabrics illustrated in figure 9. The basic fabric consists of an array of packet processors at the input and output stages, connected to three cascaded banyan networks: first a copy network which requires a switch element which can broadcast each input to both of its outputs,then an array of broadcast group translators, which modify each broadcast packet by mapping its destination to the appropriate output port address, a distribution network which relieves congestion by distributing inputs from one input port across all its output ports, and finally a

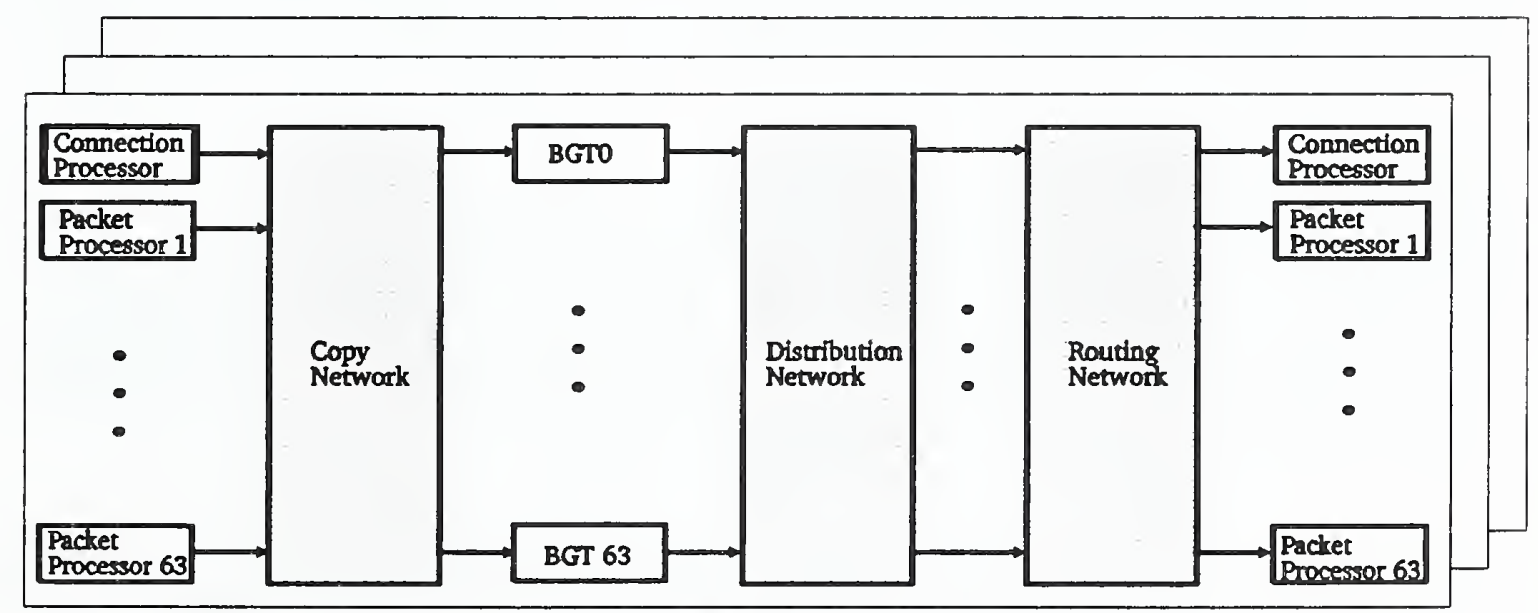

BGT: Broadcast Group Translator

Figure 9 - Turner's Broadcast Switching Fabric 
routing banyan, which then delivers the packets to the correct output port processor. An external connection processor, which communicates with other communications processors in the array through the switching fabric, determines a unique routing for each logical connection, to avoid reordering of packets.

Turner's copy network does allow blocking, and Lee [60] proposes a more elaborate copy network, with running adders to construct a nonblocking copy network. Lee also proposes adding a Batcher sorting network to the copy network, to sort packets by priority and then regulating the input packets by their priority and the number of copies requested to prevent the copy network from overflowing.

\section{Implementation of Banyan Switches}

The banyan network switch, because of its regular structure, is well suited to VLSI ICs. A many port switch is easily constructed on a single IC, by replicating the two port elements. These then may be connected on circuit cards into still larger switches. Entirely electrical banyan packet switches of considerable complexity are probably practical, although large switches become exceedingly complex. Chip interconnections can be minimized by making the network self-routing, attaching the routing to the packet in a preprocessor, and combining as many banyan nodes as possible on a single chip.

Electrically controlled $\mathrm{LiNbO}_{3}$ optical 2-input $\times$ 2-output switches are available, and it is possible, in principle, to construct large banyan optical circuit switches with these elements. In practice these elements exhibit significant crosstalk and loss, requiring periodic regeneration of the signals. A nonblocking fabric is needed, since there is no storage in the $\mathrm{LiNbO}_{3}$ switch. Since input and output packet buffering and switch control are all electrical, it is difficult to see any advantage to such a packet switch. With a circuit switch, however, the $\mathrm{LiNbO}_{3}$ banyan may be more attractive, because electronic buffering is not required and switching takes place at the full bandwidth of the optical channel. Crosstalk, loss and the need for signal regeneration in the switching fabric become limiting factors.

It appears then that banyan packet switches are likely to remain entirely electrical devices for the near future, with optical demultiplexing of any input channels whose aggregate speed exceeds the capability of electronic logic.

\section{Optical FDM Crossbars}

One obvious switching approach is to use some sort of FDM to make a completely optical crossbar. Then each input port is fed into a narrowband laser transmitter with a unique wavelength. A passive coupler combines the signals from each transmitter and the combined signals are routed to an array of output receivers. Either the transmitter or the receiver must be tunable to any of a comb of frequencies, and each of the others is fixed at one of the frequencies in the comb. Both coherent and noncoherent channel selection is possible, however a larger number of channels is possible with coherent techniques.

One advantage to the FDM crossbar is that the number of active elements is linear with $\mathrm{n}$, the number of input channels. The number of switching elements in the various banyan schemes is proportional to $\mathrm{n} \log 2 \mathrm{n}$. Another advantage is that rather high bandwidth channels, on the order of several Gbit/s, are possible.

The Hypass switch proposal for a hybrid optoelectronic switching system [4] uses tunable transmitters and fixed frequency receivers in one direction and the reverse in a parallel network in the other direction. The proposed channel rate is $2 \mathrm{Gbit} / \mathrm{s}$ and, for packets of 
1000 bits a tuning time of less than $10 \mathrm{~ns}$ is required. Collisions at output ports are possible, and a parallel coupler, with fixed frequency transmitters and variable frequency receivers, informs the transmitters of collisions. Input buffering and control processing are electronic.

Another, somewhat similar, design [119], proposes that an $\mathrm{N} \mathrm{x} \mathrm{NL} \mathrm{optical} \mathrm{switch} \mathrm{fabric} \mathrm{be}$ used. There are $\mathrm{L}$ wavelengths assigned for each output and each is buffered. A "knockout" contention controller routes up to $\mathrm{L}$ input packets to each output port. If more than L packets are simultaneously addressed to one output port, the excess packets are lost, that is "knocked-out." For $\mathrm{L}$ as small as 8 , the packet loss probability can be held below $10^{-8}$, assuming a random traffic distribution. This architecture achieves the best possible delaythroughput performance and preserves packet sequences. Like the Hypass proposal, rapidly tunable lasers are needed.

While the optical crossbar may provide an excellent switching fabric for a packet switch, very agile lasers are required. It is likely that this, and, for coherent transmission (which would increase the number of frequency channels available), the time needed for the receiver to lock onto the carrier, would require long packets for efficiency. The buffers and all control logic remain electronic, limiting channel bandwidths.

Optical frequency division multiplexing switching appears, for the foreseeable future, to be more easily adapted to circuit switching, where no electronics need intrude in the data path and electronic processing is only required at call setup time. WDM/FDM multiplexing seems, however to be so well suited to distributed switching, through a passive star network, that this is discussed in the following section as a separate topic.

\section{Conclusion}

Near term centralized packet switches are likely to be entirely electronic devices using some sort of cascaded banyan switching fabric. Hybrid electrooptical packet switches using wavelength division multiplexing appear possible, but likely would need packets longer than the 48-byte B-ISDN ATM cell, and may not offer much advantage over entirely electronic switches, depending upon the pace of development in optical components. Entirely optical circuit switches will almost certainly be practical and could switch very high rate channels, but optical WDM/FDM switching may be better suited to distributed architectures, which will be considered in the next section.

There are many complex issues in the design of large banyan fabric packet switches. Among the most vexing are bounding the variation of delay in the switches, for isochronous services such as voice traffic, in the face of contention with a highly variable, dynamic load of data traffic. Developers of such switches also will face all the packaging, cooling, maintenance and electrical chip interconnection problems of very large supercomputers, which, in effect, they are.

\section{Fast Packet Switching vs. Applications}

This section considers the suitability of the proposed B-ISDN type 48-byte cell switching for the voice, data and video applications.

\section{Fast Packet Switches for Voice}

Voice presents one particular problem for packet switches, if compatibility with the existing telephone plant is a concern. Variation in delay must be held small, even in the face of a simultaneous highly variable data load. The smaller the cell, the less the delay for pack- 
etization, and the smaller the delay usually encountered from packet congestion in switches. The small 48-byte ATM cell is the result.

\section{Fast Packet Switches for Data}

In general fast packet switching seems to suit the needs of data applications well, if the switches can be designed so that packets are not reordered and that the probability of packet loss due to switch congestion is small. There appear to be solutions, and the expense and complication of resolving them is confined to the switches.

There is one complication, however, which affects the user terminals: the 48-byte fixed cell size proposed for B-ISDN is too small. Typical computer network Protocol Data Units (PDUs) are much larger (even TCP/IP control packets will require several cells). Typical computer networks are designed to support large, variable sized packets, between 1000 to 4500 bytes. A single block of magnetic disk storage is rarely less than 512 bytes.

In recognition of this problem the Switched Multimegabit Data Service (SMDS) has been proposed by North American telephone carriers [41], [103]. SMDS will provide users a logical interface above the ATM level, supporting logical packets of more than 9000 bytes, similar in format to standard LAN protocols. This will initially be implemented on top of the IEEE 802.6 DQDB Metropolitan area Network bus service [116]. There is one complication. The terminal adaptor reassembling the logical SMDS packet from 48-byte cells may have to be concurrently reassembling logical packets from an indeterminate and potentially large number of sources at a high rate. Consider the design of an SMDS terminal adaptor for a network storage server which may be more or less simultaneously communicating with hundreds of workstations. A banyan packet switch may be needed in the end terminal adaptor for such applications.

\section{Fast Packet Switches for Video}

The fast packet switch seems a satisfactory means for switching digital video signals for long distance transmission. It is an appropriate means for point-to-point video transmission for conferencing, security monitoring and the like. Packet switching as a means for distributing video to homes seems a questionable choice. It adds a whole extra level of a copy network to the switching fabric in local office switches, and video distribution will then dwarf all other traffic. The rest of the switching fabric must also therefore be augmented to carry video. The home terminal adaptor must be capable of shuffling packets fast enough to sort out a couple of video channels, occasional voice packets and perhaps data packets, all at the same time. Either the television set or the adaptor must have enough buffer memory to compensate for any variation in the packet arrival rate. To keep the switching load, as well as the data rate down, highly compressed digital video would be transmitted for HDTV services, requiring television sets to have complex decoders with as much as two full frames of memory. 


\section{Passive Optical Broadcast Star Networks}

The term Passive Optical Network (PON) has been coined, apparently by researchers at British Telcom in the United Kingdom [90,17], to describe a very appealing network architecture which a number of researchers are pursuing in the United States, Britain and Germany. While the details and scope and applications of the various experiments and designs vary considerably, they all involve using a star coupled broadcast network and some form of frequency, wavelength or subcarrier multiplexing to allow the broadcast network to carry a number, potentially quite large, of separate high bandwidth channels. Optical processing, then, is used to combine and select channels whose aggregate bandwidth can far exceed the rate of any serial electronic circuit. Within the frequency dependent optical channels a second tier of time or code division multiplexing may be used to allow large numbers of lower rate users to share a high bandwidth optical channel. These are broadcast networks; every station sees the signal sent by every other station and must select the channel it receives and, in some cases identify by address the packets sent to it. It is passive in that signals are not regenerated in the network and no switching occurs in the network. Broadband linear amplification of signals is allowed; indeed in large networks it is needed. This is a very simple, yet powerful and flexible network structure which is made possible by the extraordinary high bandwidth, low dispersion and low loss properties of single mode optical fiber.

While frequency and wavelength division multiplexing appear to be essentially different words to say the same thing, a distinction is often made in the literature. The term frequency division multiplexing (FDM) is commonly reserved for coherent systems, which generally allow very dense spacing of channels, while wavelength division multiplexing (WDM) is used for noncoherent systems, often relying on etalon filters, which generally make much less dense use of the optical spectrum. The convention of using FDM to imply coherence and WDM to imply noncoherence will be observed here.

It has been estimated that the limitations of components will limit single channel TDM systems to a maximum of about $20 \mathrm{Gbit} / \mathrm{s}$. With WDM, a total network capacity of around $100 \mathrm{GHz}$ (out of a total spectrum of around $30,000 \mathrm{GHz}$ ) is realistic using optical filtering and direct detection [61]. This would correspond to a total bit rate of 50 to $100 \mathrm{Gbit} / \mathrm{s}$. Considerably more capacity would be available using coherent FDM. The FDM and WDM channels can be independent and have very different properties depending upon their application.

In Subcarrier Division Multiplexing (SCM), the frequency multiplexing takes place in the electrical, rather than the optical domain as illustrated in figure 10. For some purposes $(e$. g. video distribution) one might wish to combine SCM, in a two layer hierarchy, with FDM/WDM selection of an optical channel before demodulation of the electrical signal. It can also serve by itself as a multiple access technique for lightwave networks [20]. In such a SCM multiple access system, light from all the system transmitters falls on the detector photodiode, therefore the shot noise is the sum of shot noise for all the channels. This makes SCM receivers somewhat less sensitive than receivers in FDM/WDM systems. Strict control of the wavelength or modal characteristics of transmitters is not required, and the multiplexing and demultiplexing of signals is with mature, conventional electronic components.

So far, most of the experimental activity with SCM has not been for providing multiple-access data networks, rather it has been for the purpose of distributing multiple, conventional 


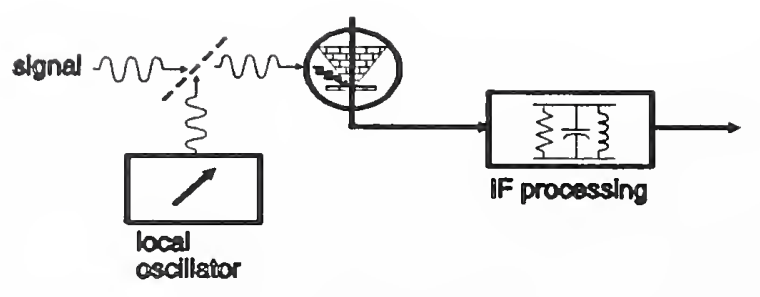

(a) heterodyne receiver

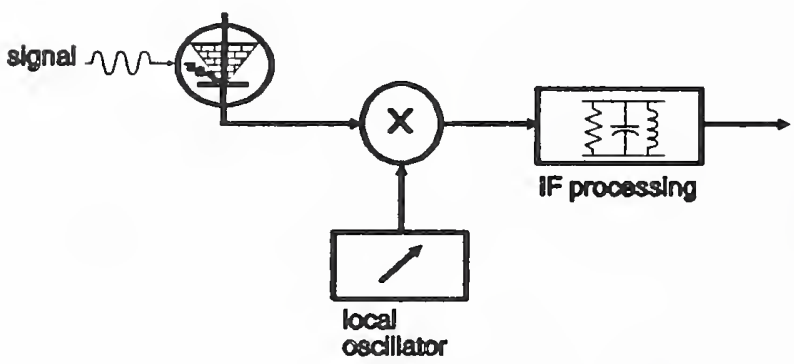

(b) SCM receiver

\section{Figure 10 - Heterodyne and SCM Receivers}

analog or digital video channels originated by a single transmitter $[108,74,53,80,36,21]$. SCM potentially provides an easy and effective way to use comparatively mature and inexpensive microwave technology to select individual video channels from an optical channel carrying a number of channels. It provides a solution of how to effectively use the huge installed base of video equipment with fiber optic distribution.

In principle, such a broadcast medium could be either a linear bus or a star. In practice it seems likely that it will be a star. Very good single mode integrated star couplers have been built, but practical " $T$ " taps, required for linear buses have proved difficult. Practical fiber bus taps tend to be unidirectional (this can be an advantage as well as a disadvantage). Each tap adds an excess insertion loss, which is not dramatically less then the entire excess insertion loss of the star coupler. When a station at one end of the bus transmits, stations at the far end see the sum of the insertion and coupling losses, and a consequently weak signal, while stations at near the transmitting station see a very strong signal. This creates a large dynamic range for receivers.

With a star coupler, however, all stations see the same major loss element, the star coupler, and the dynamic range seen by receivers is much less. Moreover, there is a single insertion loss for the coupler, which is not dramatically more then the individual losses of each separate tap in a bus system. With the very low losses of single mode fibers, particularly in the $1500 \mathrm{~nm}$ region, passive star networks with a surprisingly large number of stations spread over a considerable distance seem realizable. Of course, the star, as illustrated in figure 11, is not made of a single monolithic coupler for large numbers of stations, but a number of combined couplers.

There have been numerous demonstrations reported, showing the basic concept of broadcast star lightwave using FDM [7, 35, 34, 16], WDM [96, 58, 94, 18] and SCM [15, 80, 21]. The concept is attractive. Which of the alternatives succeed commercially and when depends largely on the commercial development of the needed optical components, which 

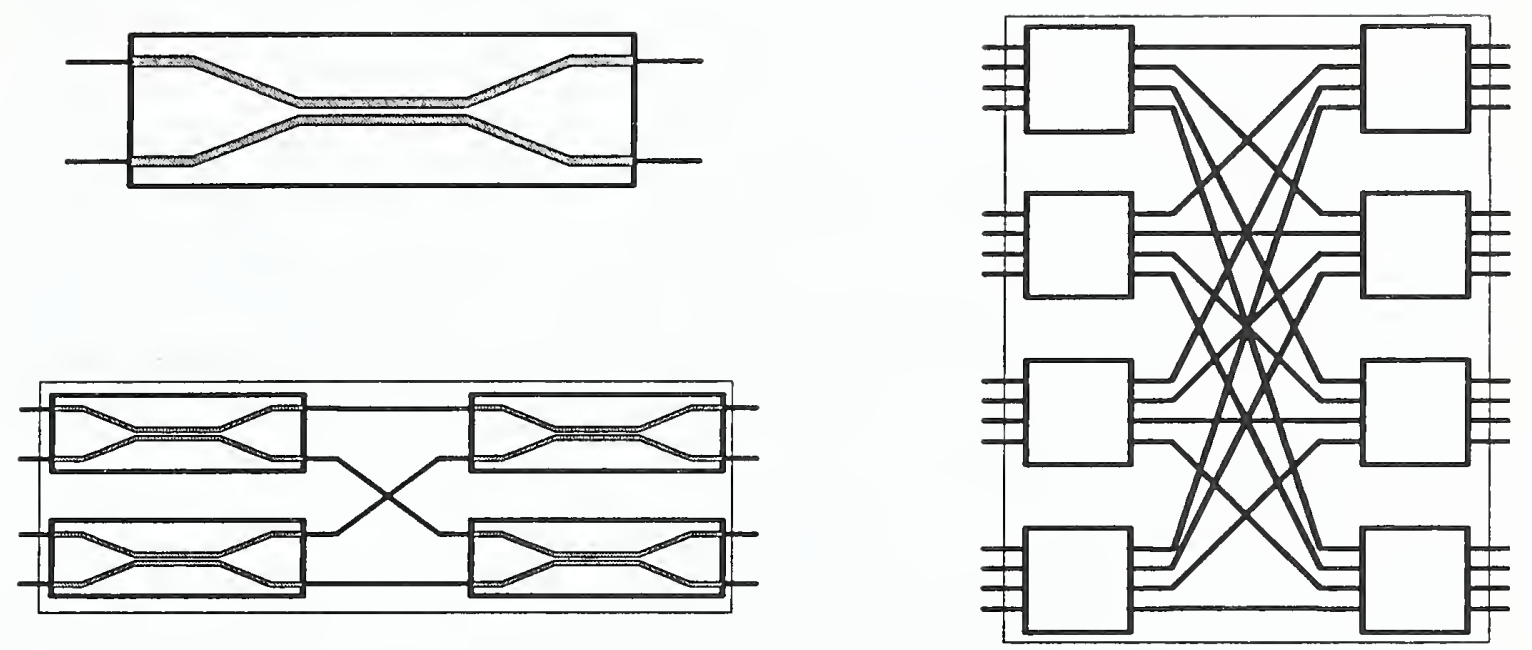

Figure 11 - Star Coupler

have been demonstrated in laboratories, but which must be reduced to off-the-shelf high volume commercial production before the PON concept can be successfully exploited.

\section{PON LANs Without Amplifiers}

How large can the PON network be without amplification? In the $1500 \mathrm{~nm}$ region a loss of $.2 \mathrm{~dB} / \mathrm{km}$ is conservative. Star coupler efficiencies of $50 \%$ seem achievable and an experimental $19 \times 19$ coupler with uniform coupling and a 38\% efficiency has been demonstrated [56]. Semiconductor lasers with a $1 \mathrm{~mW}$ output are widely available. Assuming a $10 \mathrm{~dB}$ total loss for coupler excess insertion loss plus other splicing and connector losses in links, it is possible to estimate the number of stations and the maximum radius from the star for various power budgets. Budgets from 30 to $50 \mathrm{~dB}$ seem realistic, depending upon the data rate and technology used. Coherent detection might add another $10 \mathrm{db}$ to the power budget. For example, the theoretical limit for single filter heterodyne FSK signalling (a fairly easy receiver to make, but $3 \mathrm{~dB}$ sensitive than dual filter FSK) is $-57 \mathrm{dBm}$ at $140 \mathrm{Mbit} / \mathrm{s}$, and $-50 \mathrm{dbM}$ has been demonstrated [88]. Table 1 illustrates some possibilities for maximum distance from the coupler versus the number of stations for power budgets of 30,40 and $50 \mathrm{~dB}$.

Table 1 - Power Budget vs. Network Size

\begin{tabular}{llll}
$\begin{array}{l}\text { Power } \\
\text { Budget } \\
(\mathrm{dB})\end{array}$ & $\begin{array}{l}\text { Excess } \\
\text { loss } \\
(\mathrm{dB})\end{array}$ & $\begin{array}{l}\text { Number } \\
\text { of } \\
\text { Stations }\end{array}$ & $\begin{array}{l}\text { Maximum } \\
\text { Radius } \\
(\mathrm{km})\end{array}$ \\
\hline 30 & 10 & 32 & 12 \\
40 & 10 & 256 & 15 \\
50 & 10 & 512 & 32.5
\end{tabular}

We have assumed $1500 \mathrm{~nm}$ operation in these examples. This gives us the lowest loss per $\mathrm{km}$ of fiber. It is quite likely, however, that truly local PON networks, would utilize either the $1300 \mathrm{~nm}$ region, with attenuations on the order of $.5 \mathrm{~dB} / \mathrm{km}$ or the $850 \mathrm{~nm}$ region with 
attenuation on the order of $3 \mathrm{~dB} / \mathrm{km}$. Even with a loss of $3 \mathrm{~dB} / \mathrm{km}$, if the radius to the hub is $2 \mathrm{~km}$ or less, only $12 \mathrm{~dB}$ is needed for fiber attenuation, and, at the present time, $850 \mathrm{~nm}$ devices are less expensive and offer rather better performance in several respects, particularly higher laser output powers. The maximum distance of point-to-point links is strongly a factor of fiber attenuation and only weakly a factor of the transmitter power. In a power limited link, doubling the transmitter power adds only a fixed increment to the maximum distance, but halving the fiber loss coefficient doubles the maximum distance. On the other hand, the ability to support a large number of stations in a small area with a passive coupler is primarily a function of the power budget and doubling the power doubles the number of stations. While $1500 \mathrm{~nm}$ operation will always be chosen where fiber attenuation is the major loss element, 850 or $1300 \mathrm{~nm}$ operation are feasible, and perhaps more desirable, when splitting losses are the main loss concern.

\section{The PON as a Metropolitan Area Network (MAN)}

Clearly, rather large local networks could be supported without amplification in the network. However, semiconductor linear amplifiers will make much larger networks practical. Gains of 6 to $8 \mathrm{~dB}$ from a single amplifier have been demonstrated in a 20 channel $1 \mathrm{gbit} / \mathrm{s}$ per channel passive star, with a bit error rate penalty due to amplifier spontaneous emission noise of .5 to $2 \mathrm{~dB}$ [18]. This gain is sufficient to allow a factor of 4 increase in the number of stations. Very low noise semiconductor and fiber amplifiers will likely become available. Four in-line amplifiers having a total gain of over $40 \mathrm{~dB}$ have been demonstrated at a 1 Gbit/s rate, providing an overall budget of over $70 \mathrm{~dB}$ [72]. An overall budget of $70 \mathrm{~dB}$ seems reasonable with amplifiers, but as the size of the star grows, it is likely that the excess loss will as well. Some possibilities for stars with $70 \mathrm{~dB}$ power budgets and 10 and $20 \mathrm{~dB}$ excess losses are shown in table 2 below:

Table 2. Power Budget vs. Network Size

\begin{tabular}{llll}
$\begin{array}{l}\text { Power } \\
\text { Budget } \\
(\mathrm{dB})\end{array}$ & $\begin{array}{l}\text { Excess } \\
\text { loss } \\
(\mathrm{dB})\end{array}$ & $\begin{array}{l}\text { Number } \\
\text { of } \\
\text { Stations }\end{array}$ & $\begin{array}{l}\text { Maximum } \\
\text { Radius } \\
(\mathrm{km})\end{array}$ \\
\hline 70 & 10 & 4,096 & 60 \\
70 & 10 & 65,536 & 30 \\
70 & 10 & 262,144 & 15 \\
70 & 20 & 8,192 & 27.5 \\
70 & 20 & 16,384 & 20
\end{tabular}

All the examples shown above are based on one assumption which, while reasonable in the small LAN environment, ignores one of the great advantages of the PON approach. They assume a direct fiber pair from each end terminal to the passive star hub. This provides the most favorable power budget and it is essentially how present telephone local exchanges are wired, but it maximizes the amount of fiber required. In networks covering larger areas, the amount of fiber plant needed can be dramatically reduced if part of the central star is made a tree of splitters and combiners as illustrated in figure 12. This illustrates a possible design for a 65,536 line network, covering a radius of $20 \mathrm{~km}$. This could serve the needs of a fair size city, and certainly corresponds to the coverage of a large telephone local office switch. Two stages of 16-way splitters and combiners are combined with a 256 way central splitter. The inherent loss in the network due to power division has increased by $24 \mathrm{~dB}$ (from the pure star) to $72 \mathrm{~dB}$. Assume that the excess insertion loss of the 16 way splitters and combiners is $3 \mathrm{~dB}$ and the excess insertion loss of the central hub is $9 \mathrm{~dB}$ and allow $5 \mathrm{~dB}$ for 


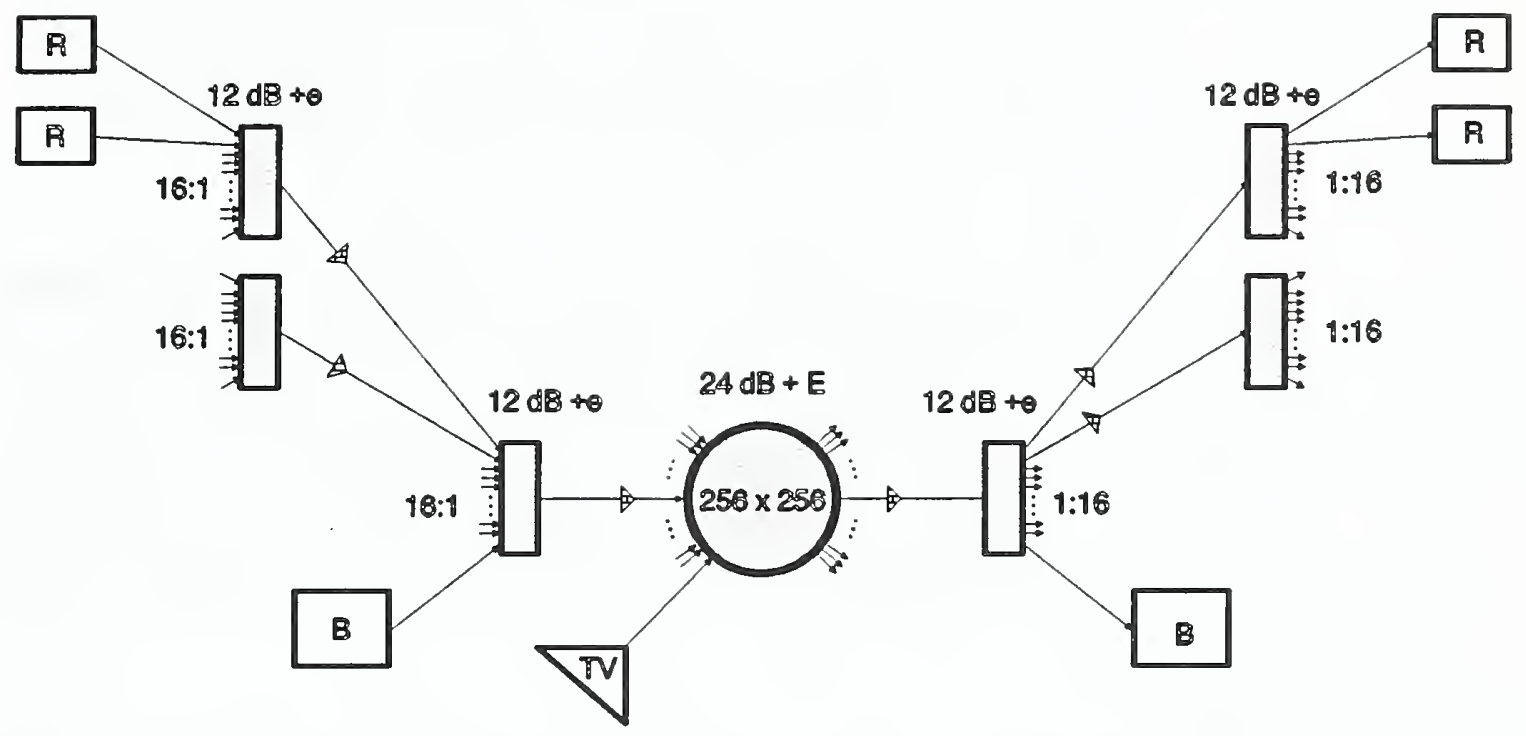

Figure 12 - The Passive Optical Network

splices, etc, giving a total excess loss of $26 \mathrm{~dB}$. Assume a $20 \mathrm{~km}$ maximum distance giving a maximum round trip transmission loss of $8 \mathrm{~dB}$. The total power budget required is $106 \mathrm{~dB}$.

The network in figure 12 has 4,608 amplifiers. If we can get $12 \mathrm{~dB}$ of gain from each amplifier (high for present semiconductor linear amplifiers, but not unreasonable in the fairly near term) the remaining budget is $54 \mathrm{~dB}$, and, if we accept the $50 \mathrm{~dB}$ budget for a 140 Mbit link, then we fall $8 \mathrm{~dB}$ short.

However, high bandwidth transmission is probably not needed from homes. There is a direct inverse relationship between bit rate and receiver sensitivity, because the power transmitted in each bit time is inversely proportional to the bit rate. Assume that residences transmit on $17.5 \mathrm{Mbit} / \mathrm{s}$ channels. We gain $24 \mathrm{~dB}$ from the reduced rate, less $3 \mathrm{~dB}$ to split receive channel power for two different receivers, plus $1 \mathrm{~dB}$ excess loss for a separate higher bandwidth video receiver. We now have a margin of $12 \mathrm{~dB}$ for home voice and data transmission services.

Broadcast video, can be injected right at the hub. For every video signal injected at the hub, 256 fewer stations could be attached to the network, but $25 \mathrm{~dB}$ would be saved in power combining losses and $4 \mathrm{~dB}$ would be saved in fiber losses for the video signal. Allowing a $4 \mathrm{~dB}$ splitter loss in the residence to split the video from the voice/data service gives a total loss of $52 \mathrm{~dB}$, leaving a $2 \mathrm{~dB}$ deficit. It is reasonable to suppose that somewhat higher power transmitters might be used for broadcast video channels. A $2 \mathrm{~mW}$ transmitter would add the extra needed power, while a $15 \mathrm{~mW}$ transmitter would have enough power for a 1 Gbit/s channel, which could provide several compressed HDTV signals, or it could provide an uncompressed HDTV signal, allowing the use of less expensive video decoders. 
Businesses needing to send data at high rates, might connect to the inner layer of splitters and combiners, costing 16 residential lines for every high bandwidth business line, but also saving $6 \mathrm{~dB}$ in power losses. If businesses could afford $2 \mathrm{~mW}$ transmitters, then power requirements for the $140 \mathrm{Mbit} / \mathrm{s}$ rate would be met. These businesses might very well operate their own local PON networks on their premises, perhaps at $850 \mathrm{~nm}$. If the local PON were coupled to the metropolitan area PON by a laser amplifier operating in the 1300 or $1500 \mathrm{~nm}$ regions, the local PON and metropolitan PON could be combined in the user premises.

The example given above takes no account of amplifier saturation effects. Since the laser amplifiers are similar to the transmitters, they may saturate at similar power levels. If all the transmitters attached to one combiner were to transmit simultaneously, the amplifier might be driven to saturation. However, this can probably be handled with suitable transmission protocols for the voice and data services; the $17.5 \mathrm{Mbit} / \mathrm{s}$ residential voice and data channels postulated above, should handle residential needs with a fairly low duty cycle. It might be that amplifiers would only be used on the splitter network, where signal levels have been attenuated by several levels of excess insertion loss and are averaged over the entire network population.

\section{Wide Area PONs}

There is no reason in principle that the PON, or a part of its bandwidth (probably the 1500 $\mathrm{nm}$ region) can not operate over very large distances. Surely areas the size of present Local Access Tariff Areas (LATA's) are in principle practical, and Cochrane and Brain [17] speculate that a single $\mathrm{PON}$ large enough to span entire countries may eventually become practical.

Each of the three windows offers particular advantages. The $1550 \mathrm{~nm}$ window offers very low attenuation. The $1300 \mathrm{~nm}$ window has somewhat higher loss, but it is easy to make fiber with low chromatic dispersion in this region, and (at least at present) the lasers are less expensive than at $1500 \mathrm{~nm}$. At $850 \mathrm{~nm}$ relatively mature AlGaAs technology results in lower costs and much higher power outputs for lasers, but fiber attenuation is significantly higher. The $1500 \mathrm{~nm}$ window looks suitable for wide area networks, while the $850 \mathrm{~nm}$ window seems well suited to densely populated local networks.

This suggests a three tier architecture, illustrated in figure 13, allocating the $850 \mathrm{~nm}$ band to local PONs, the $1300 \mathrm{~nm}$ band to metropolitan area PONs and the $1500 \mathrm{~nm}$ band to a wide area PON. Semiconductor laser amplifiers typically have a passband of about $50 \mathrm{~nm}$ and must be designed for each band using materials with appropriate band gaps. The 850 $\mathrm{nm}$ signals would not be sent out of the most local level of this hierarchy and entirely local traffic would stay in the local PON. The $1300 \mathrm{~nm}$ signals would be projected across the LAN/MAN network boundary with suitable amplifiers, but not across the MAN/WAN boundary. The $1500 \mathrm{~nm}$ band signals would be projected across both boundaries, so that signals transmitted at $1550 \mathrm{~nm}$ would be available anywhere on the combined PON.

Semiconductor (or perhaps Raman fiber) laser amplifiers appear likely to provide the gain and low noise characteristics needed for such a large, three level WAN. Assume, for example, a radius of $100 \mathrm{~km}$ and 8 million stations with a $1000 \times 1000$ splitter at the hub of the network. Then the intrinsic power budget required for signal splitting loss is $108 \mathrm{~dB}$. Only $40 \mathrm{~dB}$ are needed for intrinsic fiber loss. Allowing for splicing and excess insertion losses, etc, a total end-to-end budget of $200 \mathrm{~dB}$ might be required. If the transmitters and receivers provide $50 \mathrm{~dB}$ of this, then amplifiers must account for $150 \mathrm{~dB}$. Broadcast video 


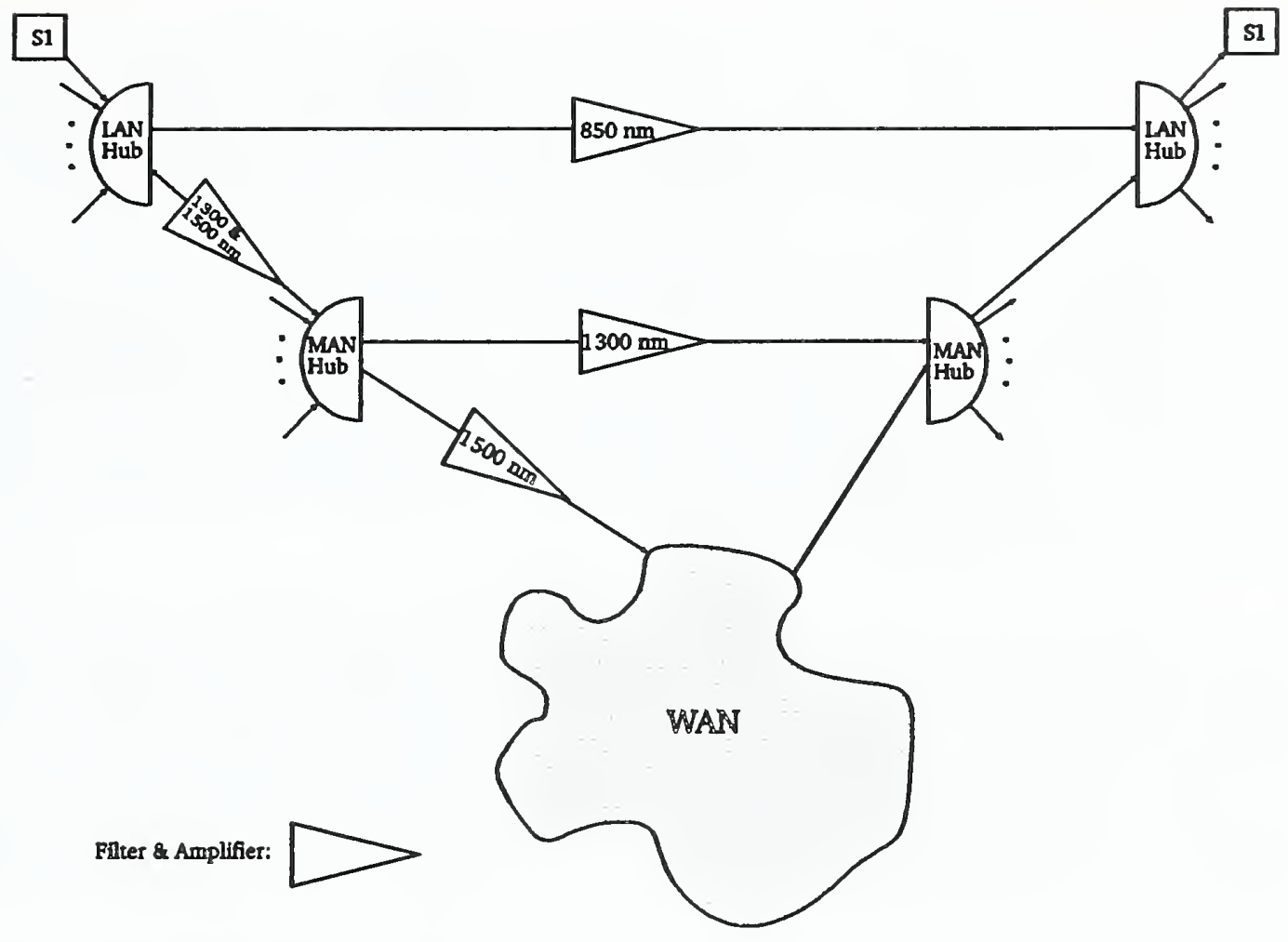

Figure 13 - Wide Area PON, Using $850 \mathrm{~nm}$ Window for LANs

signals could, as before, be injected at the hub, reducing the budget to $140 \mathrm{~dB}$ and the gain required from amplifiers to about $90 \mathrm{~dB}$. This much amplification is probably, ultimately, reasonable, but the maximum extent of such a network will be a complex tradeoff of many factors.

It may also be too optimistic to expect that effective splitters and other components can be developed, which work well over so wide a spectrum. There are commercially available splitters which work at both 1300 and $1550 \mathrm{~nm}$, and the excess insertion loss for the dual wavelength parts is often about double that for parts optimized for a single wavelength. It may prove that only a two tier $1300 \mathrm{~nm}$ and $1550 \mathrm{~nm}$ PON is practical, and that the $850 \mathrm{~nm}$ window is used for customer premises LANs which are not optically coupled to the wider area PON.

There are several other problems which have not yet been considered. The first, and most obvious, is dispersion or pulse spreading. We have neglected dispersion so far, because we have considered only fairly short distances and narrow linewidth sources. We have also neglected the possibility of using a single, very high rate $(20 \mathrm{Gbit} / \mathrm{s}$ or higher) TDM approach, because of the apparently high cost of making every receiver deal with a very high rate channel; rather we have discussed using FDM access to many slower rate channels. But as distances increase, we must consider dispersion, since we are amplifying signals, not regenerating them. The principle dispersion effect is chromatic dispersion. In $\mathrm{SiO}_{2}$ fiber chromatic dispersion typically goes to zero in the $1300 \mathrm{~nm}$ region. This might suggest using the $1300 \mathrm{~nm}$ band for the WAN signals, however the fiber loss is higher at $1300 \mathrm{~nm}$ than at $1500 \mathrm{~nm}$. We can do something about dispersion, however. By making a two or three level structure for the core of the fiber, each with a different index of refraction, a phenomenon called "waveguide dispersion" can be used to balance the chromatic dispersion in the 1500 
nm region. A "dispersion-shifted" fiber can be made with its zero dispersion point in the $1500 \mathrm{~nm}$ region A "controlled-dispersion" fiber can also be made where the two dispersion factors cancel at two wavelengths rather than one, and dispersion is near zero for a wide band [42]. If the WAN links were of controlled-dispersion fiber and coherent modulation were used (which uses narrow line width sources) then dispersion would be minimized.

The second class of effect which would be a concern is stimulated Brillouin and Raman scattering Both are very nonlinear phenomena, which manifest themselves as a power dependent excess fiber loss and crosstalk, when powers exceed some threshold level, but are negligible otherwise. Brillouin crosstalk can be minimized with appropriate channel spacings, or modulation techniques which spread the spectrum sufficiently. One analysis for relatively coarsely spaced WDM systems indicates that, if WDM signals are spread evenly over a range of $40-100 \mathrm{~nm}$, then Raman scattering will start to become a problem when the total power launched into the fiber exceeds $40 \mathrm{~mW}$ [42].

Raman scattering may impose a practical limit on the bandwidth of the fiber for WDM systems. The fundamental quantum limit to a $10^{-9} \mathrm{BER}$ is nine photons per bit. A level of $40 \mathrm{~mW}$ corresponds to a flux of about $10^{16}$ photons per second. This would seem to imply a limit of about $10^{15} \mathrm{Bits} / \mathrm{s}$ on a fiber, assuming the 9 photons per bit quantum limit. However, we could not allow the signals to approach the quantum limit in the transmission system without severe amplifier noise penalties. If we were to try to maintain a minimum of 10,000 photons/bit in the distribution network, then the total bit rate corresponding to 40 $\mathrm{mW}$ is $10^{11} \mathrm{bits} / \mathrm{s}$. If we have 50 channels, then each would be $2 \mathrm{Gbit} / \mathrm{s}$ channels.

Raman scattering is mainly a problem in the relatively coarse WDM case. It is more likely that FDM rather than WDM networks would be used for a WAN. FDM supports far more channels at denser spacings. Raman gain in $\mathrm{SiO}_{2}$ fiber increases approximately linearly with channel separation up to a separation of about $15,000 \mathrm{GHz}$ (more than $100 \mathrm{~nm}$ in the 1500 $\mathrm{nm}$ region). In a densely spaced multichannel FDM system, the total power allowed for a given Raman induced noise penalty scales inversely with the occupied bandwidth [63]. If we assume comparatively dense coherent FDM channel spacing, then much higher data rates are possible before Raman crosstalk becomes a problem.

Intermodulation distortion and crosstalk in amplifiers also becomes a concern when we hope for a total amplification of $150 \mathrm{~dB}$. This is highly dependent on the modulation technique used, the number of channels and their bandwidth and spacing, as well as the number of simultaneously active transmitters. For example, in one experiment measuring crosstalk in a 10 channel heterodyne system, with $70 \mathrm{Mbit} / \mathrm{s}$ channels spaced at a $5 \mathrm{GHz}$ spacing, severe crosstalk was observed using ASK modulation, but virtually none using PSK/FSK modulation [7], which maintain constant power levels.

There are a number of practical problems in the engineering of wide area PONs and their implementation will be challenging. However, given the kinds of devices which are likely to be available in a decade or two the problems do not appear insurmountable, for areas on the order of a medium size state. Very wide area PONs, that is PONs the size of a continent or a major country, or a region of a major country, may require either the perfection of very good optical amplifiers and dispersion shifted fiber, or, alternatively, fluoride glass fiber and components for operation at wavelengths over $2,000 \mathrm{~nm}$, so that very long distance transmission becomes practical without amplification. Certainly such networks appear to be far off and may never be practical. 


\section{Channel Access Protocols for the PON}

It is clear that the first answer to more fully utilizing the bandwidth inherent in fiber lies in frequency or wavelength multiplexing. It is also clear that it will be a long time before sources with such exquisitely narrow linewidths and great frequency stability become available (if they ever do) that we can efficiently divide the optical spectrum into a very large number of individual low data rate channels (say $64 \mathrm{kbit} / \mathrm{s}$ voice channels) to give every low data rate user his own unique frequency channel. What does seem practical is that we will be able to have a reasonable number (say 50 , initially, to perhaps 5,000 eventually) of medium to high data rate (100 Mbit/s to $5 \mathrm{Gbit} / \mathrm{s})$ channels. This optical signal processing will allow orders of magnitude improvement in the utilization of the fiber, while limiting the speed of individual channels to rates compatible with electronic devices. To allow many thousands of simultaneous low rate users, a two level multiplexing strategy will probably be needed

\section{Frequency Division Multiple Access (FDMA)}

Each WDMIFDM channel is a broadcast channel potentially available to all endpoint stations. One possible obvious approach to using an FDM/WDM for computer communications requires that each node has a tunable transmitter and a fixed frequency receiver. $\mathrm{A}$ node wishing to send to another node simply selects the right frequency and transmits. This is the classical frequency division multiple access (FDMA) protocol. If two transmitters simultaneously send to the same receiver, a collision may occur.

In general, however, it may be easier to tune receivers than transmitters, since the transmitter is inherently a high power device. In this case an additional single common channel, using fixed frequency transmitters and receivers, can be provided to call some sort of arbiter function which arbitrates channel usage. This common call establishment channel is probably a good application for an ALOHA protocol (see below). In this case collisions should be avoided (except during call establishment) and it would probably be efficient for long burst traffic, such as file transfers.

Both receivers and transmitters might be made tunable. In this case a separate channel arbitration node could be used to make channel assignments, and a large number of nodes might efficiently share a limited number of channels for bulk transfers.

However, if there are a large number of nodes, and a much smaller number of channels, which is likely, a number of nodes are likely to share the same channel. Moreover, the tunable element in stations may be adjustable over only a small part of the total spectrum, allowing access to only a part of the bandwidth. Because of the very high frequency of the carriers, even very narrow linewidth sources will have a carrier width of many $\mathrm{MHz}$ and coherent modulation will only be possible at high signal data rates. Therefore, while unique assignment of FDM channels is a possibility for very high rate applications, other channel access protocols will be needed in combination with FDMA to allow many stations to share the same comparatively high bandwidth channels, and FDMA will often be combined with other access protocols.

\section{Channel Sharing Protocols}

In effect, by using FDMA, we have reduced the individual channel rates of a very high bandwidth network, to a more manageable lower level. However another level of multiple access protocol will be needed to accommodate very large populations of moderate to low rate stations, on the comparatively few higher rate FDMA channels. The basic choices boil 
down to some form of time division multiple access (TDMA) or code division multiple access (CDMA) protocols.

\section{TDMA protocols}

There are a variety of TDMA protocols, some of which, such as the familiar Carrier Sense Multiple Access with Collision Detection (CSMA/CD) and Aloha are probabilistic in nature and may show adverse effects under high loads. Others TDMA protocols, such as the various token bus and synchronized time slot reservation protocols have a higher overhead, and longer delay under light load, but are more predictable and sometimes more efficient characteristics under heavy loads.

Note that the situation in a PON may become similar to VSAT networks serving multiple earth stations. In both cases, propagation delay is likely to be long compared to message transmission times. Carrier Sense Multiple Access (CSMA) protocols are of limited utility, unless typical transfers are very long. Multiple access protocols for satellite transmission have been widely studied, and include the well known ALOHA and slotted ALOHA protocols, as well as various reservation TDMA protocols [112], [78]. The simplest of these protocols is the pure ALOHA protocol. A node having a packet to transmit immediately sends it. If it arrives at the hub at the same time as another packet, a collision occurs. If the transmitter can receive on the same frequency, it can detect any collisions. If not, collisions must be detected by a time-out mechanism. The efficiency of ALOHA is low (18\% max) for a large population of nodes and random traffic.

Stability is a problem with ALOHA. However, for predominantly interactive traffic, with variable length packets and a large number of low duty factor users, it can be efficient. There may be many such applications, and with very high potential bandwidths, and many channels, it may be quite possible to make effective use of ALOHA or similar protocols in PON Networks.

Strict time division multiplexing of fast channels is also possible. A single time reference channel can synchronize a number of TDMA channels. Each station can determine the delay to the hub by timing its own transmission. A central controller can allocate TDM slots to a station, and that station arranges its transmission time so that the packet arrives at the hub at the proper time, using the synchronization channel as a reference. Such a structure is more complex than Aloha, but also very efficient.

If we use comparatively low rate transmitters over a restricted distance, then various CSMA protocols may be useful. For example, the round trip delay for the PON illustrated in the section above, with a maximum distance of $20 \mathrm{kM}$ from the hub, is about $1.3 \mathrm{~ms}$. At the proposed $17.5 \mathrm{Mbit} / \mathrm{s}$ channel rate given above, that represents a delay of about 23,000 bits, which would be a $1 / 3$ second voice packet. Techniques, such as code division multiplexing, however, might create a number of slower rate channels in the higher bandwidth channel, which might then use some variation of CSMA protocol.

\section{Spread Spectrum Techniques}

Spread spectrum techniques have been intensively developed for radio communications, particularly military radio communications since the mid 1950's. They offer many advantages in military communications, including relative immunity to jamming and interception as well as multipath and the ability to allow a number of users to share a single channel without interference [83]. 
It is the last property that is particularly of interest in optical networks, because it provides an attractive, simple and potentially robust answer to the question of how very many low bandwidth users might simultaneously share a limited number of high bandwidth fiber channels in a PON. Secondary benefits include the difficulty of intercepting spread spectrum signals (without knowing the specific channel code) and, with some schemes, relative insensitivity to the drift of transmitter wavelength.

Pickholtz, Schilling and Milstein [76] provide an introduction to the general theory of spread-spectrum communications. Spread-spectrum techniques do not provide a particularly efficient way of using the available bandwidth (unless we expect to be jammed), but efficient use of the fiber bandwidth is not an issue.

Code-Division Multiple Access (CDMA) is the term given to techniques for allowing a number of transmitter-receiver pairs to use independent random modulation to share the same bandwidth, while minimizing interference. The method which is most commonly proposed for fiber optic networks is to do the spreading in the time domain [82], [77]. In this form of CDMA a bit from each source is sent as a unique pseudo-random sequence of short duration bits, in which each sequence can be distinguished from a time-shifted version of itself and every other sequence in the set. In the optical case, the required codes are somewhat different than their electrical equivalents, because conventional electrical codes take advantage of the ability of +1 and - 1 electrical signals to sum to zero, however suitable optical codes have been developed.

A single pulse is split into a staggered series of pulses by feeding it into a splitter, an array of delay lines of different length, and then combined into a single signal, as illustrated in figure 14. The signal can then be detected with a correlator which is effectively an inverse version of the coder. Detection can be by either the threshold of the combined correlated pulse or with an AND gate. If suitable optical AND gates could be constructed, then data

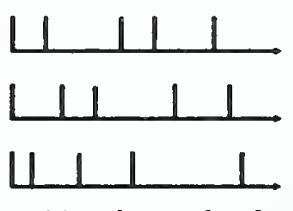

(a) orthogonal codes

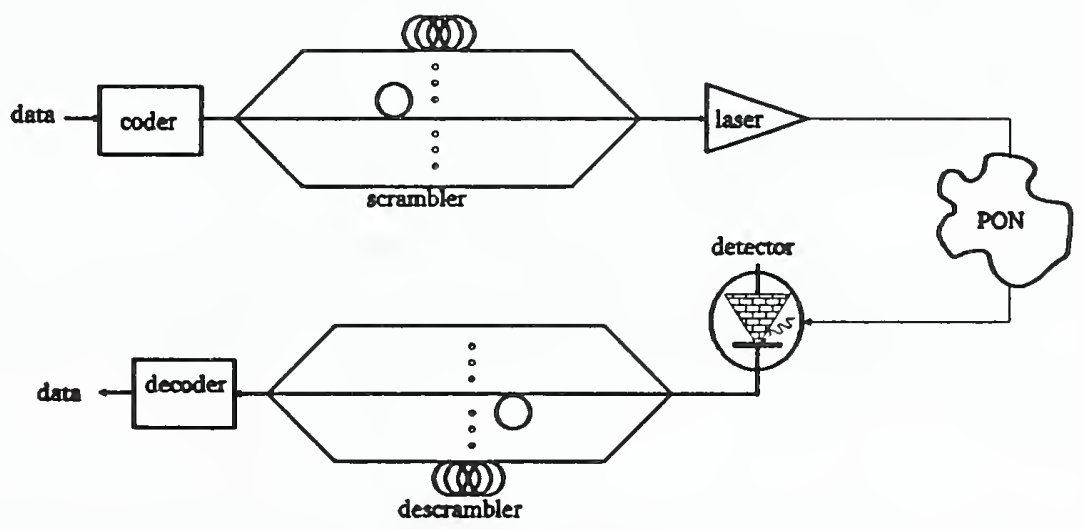

(b) CDMA in the electrical domain

Figure 14 - Code Division Multiplexing 
rates could be very high. It seems obvious that, for lower data rates, such as those required for voice, coding and decoding might be accomplished electronically, and, indeed, electronic pseudorandom sequence generators can be built up to bit rates around $1.5 \mathrm{Gbit} / \mathrm{s}$, using standard GaAs technology [104]. In such systems single mode-locked laser may synchronize the whole network, supplying a stream of very short duration pulses, to which transmitters and receivers are locked. Since some crosstalk is inevitable, the CDMA data is usually protected by a forward error correcting code.

In Random Carrier (RC) CDMA, the spreading is accomplished by the pseudorandom rotation of the phase of the modulation, according to the pseudorandom bit sequence used by the intended receiver [31]. $\mathrm{LiNbO}_{3}$ crystal phase modulators placed after the transmitter and before the receiver are used for the spreading and despreading of coherent transmissions. The primary benefit of this is that transmitter frequencies are allowed to drift randomly over a large range, eliminating the difficult problem of precise frequency control required by normal FDMA networks.

Vannucci [104] proposes that spectrum-spreading and limited control of transmission frequency be combined to improve the performance of RC CDMA. Present day DFB lasers are tunable over a comparatively narrow range via temperature control (on the order of several hundred $\mathrm{GHz}$, which is small compared to the available spectrum). Their center frequencies are not easily controlled during manufacture. But the limited tunability of the lasers might be exploited in combination with CDMA, to allow dense spacing of channels, without requiring that a precise frequency be maintained. In this system each channel monitors degradation in performance caused by crosstalk from adjacent channels and adjusts its frequency position to minimize such degradation. The interference immunity provided by CDMA allows two signals to cross each other without loss of communication, while the adaptive FDMA-like mutual avoidance mechanism limits the amount of spreading required.

Vannucci [104] proposes providing two services, a high bit rate service at $45 \mathrm{Mbit} / \mathrm{s}$ and a lower rate service below $5 \mathrm{Mbit} / \mathrm{s}$. The high bit rate service requires an electrooptic modulator at both the transmitter and receiver for spreading and despreading. Tunable Fabry-Perot filters are used at receivers in combination with DFB laser transmitters with a limited tuning range and $\mathrm{GaAs}$ pseudorandom sequence generators operating at $1.5 \mathrm{Mbit} / \mathrm{s}$, and a 4-bit-soft-decision rate $1 / 3$ Viterbi decoder with a constraint length of 6 , providing forward error correction, which can accommodate rates over $100 \mathrm{Mbit} / \mathrm{s}$ with present technology. Although the sequence generator operates at $1.5 \mathrm{Gbit} / \mathrm{s}$, it, of course must be much faster than the data rate, and the intended channel data rate is $45 \mathrm{Mbit} / \mathrm{s}$. A Mach-Zehnder modulator is used to provide binary phase shift values of $+1,0$, and -1 to modulate the on-off-keying data and to despread the signal before the receiver.

The lower rate service utilizes direct modulation of the transmitter by the sequence generator and post electronic processing of the received signal. The signal is not "despread" as such, rather sparse codes as described by Salehi [82], are used and the received signal is compared to a template of the transmitted values, and the value of the bit is determined by pattern matching.

Quite sophisticated CDMA coding techniques, whose basic principles have already been well developed for military radio applications, can be used to overcome the shortcomings of optical components, trading some bandwidth efficiency for relaxed optical component re- 
quirements. They have the very desirable properties of robustness in the face of various sources of interference and noise and little or no channel acquisition delay.

\section{Multihop Networks}

Multihop networks have been proposed as an easy way to utilize the bandwidth potential of optical fiber [1], [2]. In the multihop network nodes are connected to 2 (or more) inputs and 2 (or more) different outputs in a perfect shuffle network [117]. In the general case there are $\mathrm{N}=\mathrm{kp}^{\mathrm{k}}$ nodes, $(\mathrm{k}=1,2,3 \ldots, \mathrm{p}=1,2,3 \ldots)$ arranged in $\mathrm{k}$ columns of $\mathrm{p}^{\mathrm{k}}$ nodes. Each node has $p$ inputs and outputs. If there are 2 inputs and two outputs for each node, then $\mathrm{n} 2^{\mathrm{n}}$ nodes can be arranged as a cylinder of $\mathrm{n}$ columns of length $2^{\mathrm{n}}$, and the last column is fed back to the first, as illustrated in figure 15. Each node, receiving packets on one of its inputs, intended for another station, then selects the appropriate output path. A packet can be routed from any node to any other in not more than one circulation through the shuffle network, however blocking can occur (or nodes can be lost), and alternative routes involving more than one pass can be used. If alternative (i. e. non minimal distance) routing is allowed, then packet order can be changed; alternatively buffering can be provided in the nodes to minimize the possibility of packet loss in the event of blocking, preserving packet ordering. The performance of multihop networks has been studied and found to hold up well in the case of nonuniform loads [24].

Multihop networks can obviously be made using separate fibers for direct point-to-point connections, however, they can also be created in a PON by assigning a separate wavelength/frequency channel for each link. This allows $\mathrm{kp}^{\mathrm{k}}$ nodes to communicate, while

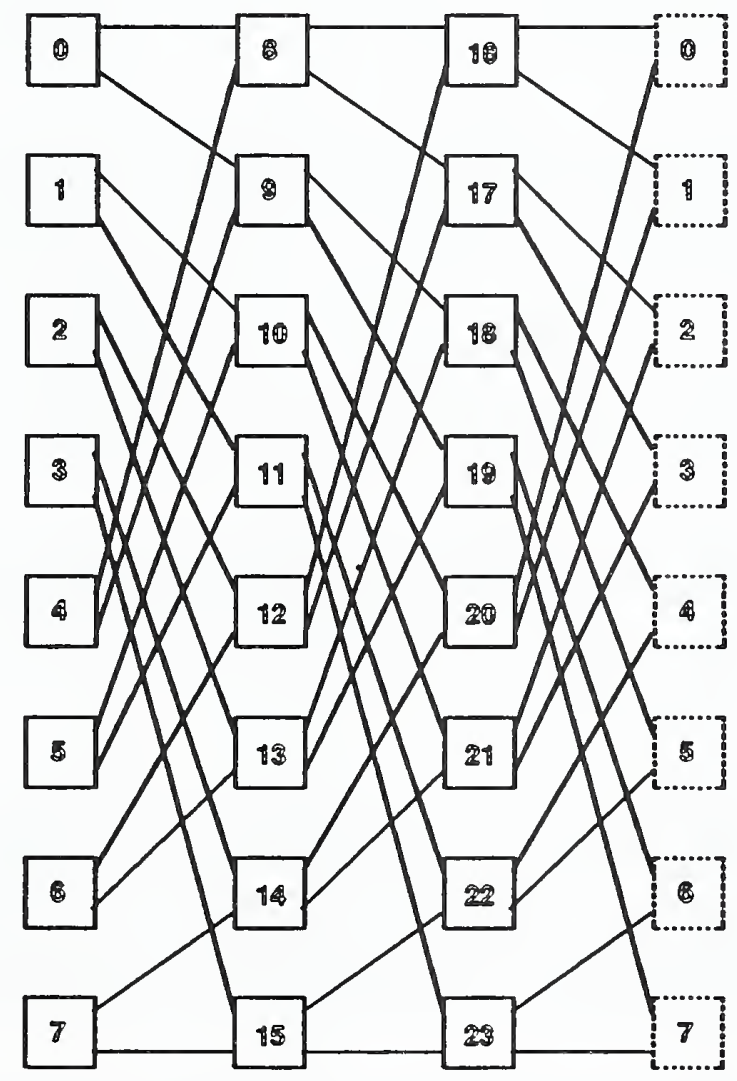

Figure 15 - The Multihop Shuffle Network 
each node is assigned $\mathrm{k}$ fixed frequency (or code) receive and transmit channels; no tuning of either transmitters or receivers is required. It is not required that all the links be on the same PON and some of the links might even be conventional point-to-point connections, while others utilize the PON. Moreover, other techniques, such as CDMA, might be utilized, in conjunction with wavelength channels, to make multihop links on a PON. Multihop, then, provides an easy and effective way to get started with wavelength division PON data applications, even without any tunable elements.

\section{Passive Optical Networks vs. Applications}

This section considers the general suitability of the PON broadcast star network for the voice data and video applications.

\section{PON Networks for Voice}

This is probably the most difficult application for the PON network, because there is already a mature, generally satisfactory circuit-switched voice network, which the PON must displace if it is to take over the voice role. During the transition period from the copper circuit switched voice network to the PON distribution network, the service provided must be compatible with the existing network. This means that delay should be more strictly bounded than would otherwise be necessary, to avoid echo problems with old equipment.

The voice bandwidth is almost inconveniently low for fiber distribution. There is no hope of efficient FDMA access of individual voice channels, the linewidths are too small compared to optical frequencies. A number of voice users must be directly combined on one much higher bandwidth channel by any of a wide variety of TDMA or CDMA techniques, so that hundreds or even thousands of voice users share a common frequency channel.

The economic justification for a PON is not likely to come from the voice application. Rather, it will come from the data and video applications, to which it is very well suited. Once PONs are widely installed for these reasons, the cost of maintaining a separate distribution network for voice, when it could be accommodated, at little extra network cost, on the PON, may eventually cause the PON to supplant the conventional copper voice distribution network. In an intermediate step, the clusters of existing twisted pair voice instruments could be connected to the PON at the block or neighborhood level sharing the cost of attachment to the high speed PON channel, which is initially likely to be comparatively high, between many users.

Due to the highly transparent nature of the PON, the present voice carrier structure, including the DS-1 and DS-3 rate multiplexed services can easily be transported, as can the proposed SONET carrier structure. During any transition period it would be easy to preserve services utilizing these carriers.

\section{The PON for Data}

The PON has great flexibility for data services, from low data rate packet transaction protocols, utilizing ALOHA, CDMA or TDMA protocols as appropriate to share a higher bandwidth broadcast channel, to point-to-point reservation of dedicated high-bandwidth FDMA channels for fast transfers of large files. A wide variety of different data services are possible at various rates depending upon the needs of the application; all are not forced into a single size of packet.

The PON can also be begun as a small LAN, and grown incrementally to a MAN as needed. It is likely that the PON concept will be first developed as a vehicle for private 
LANs and MANs, which, can, in time, grow to regional or even national size. Each separate PON need only be connected by a single fiber pair, with amplifiers as needed.

The PON offers data users a common fiber network, and a single point of attachment, for every data service. The services carried by this network can grow on an as needed basis, whether originally anticipated or not, usually without affecting other services, and without changing the fiber network itself. It's ultimate bandwidth potential is very large. Since there is no storage and no routing within the network, as with LAN networks, the network can be highly reliable and will not create duplicate or out of order packets. Of course there will be practical limits to the size of a PON, although they may be quite large, and when data communications cross PON boundaries, there may still be the possibility of duplicated or out of order packets, depending on the routing or bridging technique used.

\section{The PON for Video}

As a medium for local video distribution to residences, the PON seems to have a clear and obvious advantage over the fast central packet switch. In most respects Video is inherently a broadcast service. Present video distribution systems can be accommodated on the PON, almost without alteration. It is hard to see how any terrestrial video distribution system can succeed economically without encompassing and subsuming the present CATV distribution system and serving the huge installed base of television sets; the PON does this naturally and easily with SCM. Indeed, it seems likely that metropolitan area PON networks will first be introduced as backbone carrier networks for CATV distribution, initially with conversion to coaxial cable for the final stage of distribution, particularly where cable is already installed. With new construction, or later, as more services become available, the fiber can go all the way to the residence.

The nature of future HDTV services is far from clear. Digital transmission is an advantage in the long distance distribution network where the signal is repeated many times and the accumulation of noise can be controlled, but is probably not necessary in the lowest levels of distribution. Very high quality video distribution to residences can be provided by either analog or digital means. There are significant advantages to analog local distribution of HDTV:

- The television receiver itself will cost less since a complex, high speed digital decompression decoder is not needed.

- The bandwidth required for transmission is much less (even when extensive compression is used), allowing the same format to be used in bandwidth/power limited media such as radio or direct satellite broadcast.

- Since the bandwidth required is much less, more can be stored on recording media. Home digital audio tape or disks are one thing, but digital HDTV tape, with more than two orders of magnitude more information to store, is quite another. Digital home recording of HDTV will doubtless be practical at some point, but when? It seems unlikely that any format which cannot be cost effectively recorded at home on tape or disk will gain much acceptance.

- Formats compatible with current broadcast standards may be practical, allowing reception, with reduced quality, on existing sets and greatly easing the transition.

Digital recording of masters of programming material also allows copying without degradation, and facilitates digital signal processing for image enhancement, but is not necessarily the cost effective publishing medium. If totally digital local distribution of HDTV is 
deemed most effective, the PON is still the most suitable medium, because of its broadcast nature. Most discussion to date about digital HDTV formats has been about how to compress it, so as to minimize the bandwidth required for transmission and switching. It is far from certain, for example that the proposed $140 \mathrm{Mbit} / \mathrm{s}$ B-ISDN compressed HDTV signal is the most desirable alternative. The relative abundance of bandwidth in the PON, offers the possibility of using bandwidth to simplify sets. It might well be better to use an uncompressed $1 \mathrm{Gbit} / \mathrm{s}$ channel (which will occupy little more of the PON's channel space than a $140 \mathrm{Mbit} / \mathrm{s}$ channel). The cost tradeoffs between a much simpler but faster decoder, with little memory, and a complex decoder with a slower front end and a great deal of memory are not obvious. Much higher bit error rates could be tolerated in the $1 \mathrm{Gbit} / \mathrm{s}$ system, and the bandwidth would not be a distribution problem in the PON. A $1 \mathrm{Gbit} / \mathrm{s}$ HDTV channel would, however, make a dramatic difference in the switching load in a packet switch local distribution system.

Indeed, the abundant bandwidth of the PON offers the appealing possibility of sending the same programming in several formats. For example, assume that programming material is distributed through the long distance network in the form of Red, Green, Blue coded digital data. For the NHK studio standard, this is about $1.5 \mathrm{Gbit} / \mathrm{s}$ of data. Within the metropolitan area PON this is then coded and simultaneously broadcast in several of the following possible formats:

- NTSC (with possible compatible quality enhancement features).

- NTSC with the chrominance separated from the luminance (as is now done with Super VHS), and perhaps other enhancements providing quality improvements greater than possible with totally compatible NTSC, but requiring little extra expense when added to an NTSC receiver.

- Full resolution analog RBG HDTV with three separate $30 \mathrm{MHz}$ analog color signals, for those with displays capable of the full HDTV resolution. The display might be expensive, but the electronics would be very inexpensive, since little electronic processing would be required at the set. The cost of high speed digital to analog conversion would be shared by all sets in the PON, and a complex decoder for compressed digital would not be required. Some enhancements might be generated at the PON broadcast point, for example conversion to 60 fields per second, doubling the bandwidth requirement, but reducing screen flicker.

- Completely digital HDTV, allowing the viewer to receive the studio standard (or possibly some compressed form of it) with the generally noise free characteristics of digital transmission. If desired, the receiver could perform whatever enhancements (perhaps line doubling, conversion to 60 field scanning and the like) to the studio signal which may be desired and practical. This might be used, for example, to provide the highest possible quality display in theaters.

The PON, then, is extremely flexible and offers many exciting possibilities for video distribution, as well as providing natural support for the current installed base of television sets. The very high available bandwidths and ability to send analog signals can be used to concentrate expensive elements, such as high speed analog to digital conversion and decompression (if needed), reducing the cost of the multitude of video sets. Up until now, a great deal of effort in video distribution has always gone into minimizing bandwidth requirements; the PON and optical fiber offers the exciting possibility of using transmission bandwidth extravagantly to minimize the cost of very high performance sets. 


\section{Comparison of the PON and Fast Packet Switch}

It seems likely that it will be possible to realize either the broadcast passive optical network model or the centrally switched model of very high bandwidth integrated services networks. It is not easy to predict which can be realized the most quickly. The PON can begin as a comparatively small, simple network, and be incrementally extended, all but the simplest versions require that what are now laboratory devices be perfected commercially. The fast packet switch is inherently a complex and massive (but regular) structure, which can be made with existing semiconductor technology. Assuming that both can be realized in the about the same time frame, which is the better architecture?

\section{Passive Optical Network}

The PON has the following apparent advantages:

- The public distribution network becomes a simple, comparatively inexpensive facility.

- Maintenance of the public distribution network will be comparatively simple.

- The network can grow incrementally, starting with two nodes and investment in equipment for new services is made as needed.

- No additional switching capacity need be installed before additional users or services can be added.

- Users directly bear the bulk of the capital costs for new services as they add those services.

- Completely new services can be added simply by allocating a part of the broadcast spectrum to them.

- A PON, being logically simple, should also be highly reliable.

- Any attachment point of the network has simultaneously available all the services of the network, without any reconfigurration of the network.

- Even the front end of terminal equipment need only be designed for the minimum rate required for the intended service.

- All value added service providers have the same access to the network; there is no unfair advantage to the carrier or any provider.

- The flexibility of the medium will facilitate innovation.

- The large packet sizes, natural to computer systems, are also natural for the PON.

- The PON is a natural way to distribute video, in both current and future formats, placing few restriction on the nature of new services, easily accommodating current video services and HDTV.

Disadvantages include:

- New billing concepts will be required for network use, since there is no central control of network use (many users may simply be passive receivers). 
- Since any tap on the PON can access all traffic on the network, privacy is a concern and good encryption will be a necessity. This, however, is not very different than present use of satellite and microwave channels.

- Protocols must be defined for effectively accessing the broadcast medium and dealing with contention on access channels.

- The speed of light, and other distribution constraints as well as social and political considerations, will probably limit each separate PON to at most a region of the United States, or perhaps an area roughly comparable to Local Access Tariff Areas (LATAs) or area codes. These separate PONs must be interconnected as well.

- The public PON itself is probably a natural monopoly, since competing PONs would probably destroy the concept of universal attachment.

- The minimal switching and value added in the public network itself run counter to all the desires of the current public carriers.

- Even a bandwidth of 30,000 to $50,000 \mathrm{GHz}$ is not unlimited.

- A number of the required devices are still experimental in nature.

- Any service which requires central control would be more difficult to implement.

\section{Central Packet Switch}

The advantages of the centrally switched architecture include the following:

- It draws on the experience and tradition of current public telephone networks.

- It provides a facility for central control and billing for services used.

- Privacy is enhanced because the switches control what is visible at the terminals.

- The granularity between local, regional and truly remote services need not be as distinct as they would likely be with the broadcast architecture.

- There is no inherent limit to the aggregate bandwidth of the network.

The disadvantages include the following:

- Large high performance packet switches will be very complex devices, requiring long, expensive development, while their costs will be shared by a diminishing number of switching nodes.

- A substantial initial investment is required for switches in the network, yet this investment must be made in advance of its use for new services such as HDTV.

- Much of the rational for the high performance packet switch technology seems to hinge upon HDTV service, yet there are effective ways to provide this service. Distribution of broadcast video will swamp all other applications at the lowest level of local distribution. 
- Limitations of local office packet switches are likely to force the choice of a suboptimal format HDTV, increasing the cost of television sets and retarding acceptance.

- Public communications carriers are historically very reluctant to make large initial investments for services such as HDTV, when the market is uncertain and the return is likely to be delayed.

- The granularity of switching capacity and reliability considerations will probably require that substantial excess capacity be initially installed to allow for growth and peak demand.

- When switching capacity growth is underestimated, there may be comparatively long delays before capacity can be added to meet unanticipated needs.

- New services, not originally anticipated, may require substantial changes to the switches, or may be fundamentally incompatible with the switching plant.

- If the network provider is allowed to offer value added services, then his advantage in access to the switches may provide an unacceptable advantage to other value added service providers.

- Maintenance of complex high performance switches is likely to be expensive.

- The delay characteristics of complex packet switches and the constraints of voice systems will apparently force the partitioning of packets into unnatural units (e. g. 48 byte ATMs) for computer data services, complicating the end-to-end protocols and possibly restricting end-to-end performance.

- Selectively switched distribution of inherently broadcast very high bandwidth services such as video programming, where many users are simultaneously receiving the same channel, would add a great deal of switching complexity to the network.

\section{Conclusions}

The PON approach recognizes and takes advantage of the fundamental change to be wrought by fiber optics - bandwidth will be abundant. Therefore there are decisive advantages favoring the PON concept for future local and metropolitan area high bandwidth, integrated service networks, and perhaps for wide area networks as well. There are a host of issues and design decisions which must be made before the PON concept can be fully exploited, but one of the great virtues of the concept is that much of this can be done incrementally. The only real barrier is the reduction to high volume manufacturing of devices whose feasibility is already amply demonstrated in laboratories. The basic concepts are simple and much of the necessary theory was worked out many years ago for radio applications.

The rival approach of fast packet switching has the support of much of the world's telephone industry, and certainly the telephone switch vendors. Serious attempts will doubtless be made to realize B-ISDN fast packet switch technology, and there is little reason to doubt that practical switches can be developed. These switches require no new device technology (unless they are to use photonic switching - which does not seem likely in the first generation), but will be a massive undertaking because of their scale and complexity. If only modest, incremental improvements were to be expected in fiber bandwidth in the next generation of fiber-optic equipment, then it would be an excellent match to 
future fiber optic transmission technology. For local and metropolitan area distribution, by the time it arrives, B-ISDN will be a good solution to yesterday's problem: an efficient, flexible way to use a limited bandwidth transmission medium. But, at least in the local and metropolitan area environments, bandwidth need not be a limited or scarce resource, unless we artificially make it so.

In the wide area environment, bandwidth may be a restriction for a longer period, and there may be time for fast packet switched B-ISDN to establish itself successfully. It may be some time before the components will be available to allow truly huge PONs, spanning thousands of $\mathrm{km}$. Over very long distances, bandwidth is likely to be restricted for a much longer period. Thus a hybrid system is quite likely, with fast packet switching, and conventional circuit switching (e.g. SONET) in use for long distance transmission, while the PON dominates the local and metropolitan areas. Indeed, it is not clear when, if ever, very wide area PONs will be practical, and there must be some size beyond which they will remain impractical. It is not clear that the B-ISDN packet service is the best way to connect metropolitan area PONs, or some other strategy. For example, bridging of some sort between adjacent metropolitan area PONs in a fashion analogous to present LAN bridges, or some combination of that and long distance packet and circuit switches, is most advantageous.

Nor will the copper voice subscriber network be easily displaced for "Plain Old Telephone Service" (POTS). Well established data transmission technologies and facilities tend to remain in use far beyond the point of obsolescence. Thus, twenty years or so in the future, while we might well find fiber subscriber metropolitan area PONs widely used for video distribution and data applications, as well as some voice (particularly trunking private voice between different sites of the same organization), the copper voice subscriber network may still be the primary vehicle for residential POTS. For long distance transmission the separate metropolitan area PONs will probably be interconnected by various means, including direct bridging between adjacent networks owned by the same organization, B-ISDN ATM services, SONET circuit switched services and satellite services. As time goes on, it will be more cost effective to add the POTS service to the PON and dispense with the expense of maintaining a separate copper voice subscriber network.

It may not be too difficult to accommodate the short ATM packets, which B-ISDN will use, in the various multiplexing schemes used in the lower tier of the PON multiplexing hierarchy to share higher bandwidth frequency/wavelength multiplexed channels. Therefore, for data services, an apparently seamless interface between a PON local distribution network and a fast packet switched long distance network, is a possibility. At the same time, the long variable length packets used in present LANs are likely to be so pervasive for data applications, that there will be no substantial base of user data equipment which uses ATMs, and no need to project them into the local distribution PONs.

The format for distributing video to residences should be designed for compatibility with existing video equipment and to minimize the cost of HDTV sets. This almost certainly means that it will not be based on $140 \mathrm{Mbit} / \mathrm{s}$ compressed video packaged in ATM cells, but rather on either an analog signal or a much higher rate digital signal, intended to simplify the decoder and set. Long distance transmission may be by the $140 \mathrm{Mbit} / \mathrm{s}$ B-ISDN channel, but conversion to the uncompressed format could take place once for the entire PON, rather than every set. 
The PON, then, will start small, as private LAN or MAN for data and video, and as a vehicle for CATV distribution. PONs will eventually grow to encompass at least entire metropolitan areas. B-ISDN packet switches will naturally find their home in the long distance arena, where efficient use of bandwidth is more meaningful, where they may supplement or eventually supplant conventional TDMA circuit switches. B-ISDN ATM packet services can be brought all the way to subscribers on the PON, along with many other services. Whether the ATM becomes an important direct subscriber data interface is uncertain, since it must contend with already established IEEE 802 data packet formats, better suited to most data needs. The ATM is unlikely to become a vehicle for direct distribution of video to subscribers, because there are simpler and better alternatives.

Finally, it should be noted that these conclusions are based on the competitive comminications market which exists in the United States, and the forces which operate here. In the United States video broadcasting and communications services are provided by private companies which compete with one another to a greater or lesser degree. The same conclusions may not hold in much of the rest of the world, where both video broadcasting and telephone services are often government monopolies. Government telephone and video monopolies could, perhaps, impose solutions which subordinate video to the telephone system, but which would not be likely to survive in the more competitive United States environment. It is not necessarily considered desirable in much of the world to offer viewers the choice and range of programming selection to which Americans are already accustomed. On the other hand, there are efforts to open up broadcasting and even to make telephone companies private in some parts of the world, particularly in Britain and western Europe, which might lead to conclusions similar to those in the United States. 


\section{References}

[1] Anthonhy S. Acampora and Mark J. Karol, "An Overview of Lightwave Packet Networks," IEEE Network, January 1989, pp. 19-41.

[2] Anthony S. Acampora, Mark J Karol and Michael G. Iuchyi, "Terabit Lightwave Networks: The Multihop Approach,” AT\&T Technical Journal, Vol. 66, No. 6, pp.21-34.

[3] Gary J Anido and Anthony W. Seeto, "Multipath Interconnection: A Technique for Reducing Congestion Within Fast Packet Switching Fabrics," IEEE Journal on Selected Areas in Communications, Vol. 6, No. 9, December 1988, pp.1480-1488.

[4] Edward Arthurs, Matthew H. Goodman, Haim Kobrinski, and Mario P. Vecchi, "HYPASS: An opteoelectronic Hybrid Packet Switching System," IEEE Journal on Selected Areas in Communications, Vol. 6, No. 9, December 1988, pp. 1500-1510.

[5] E. Arthurs, J. M. Cooper, M. S. Goodman, H. Kobrinski, M. Tur and M. P. Vecchi, "Multiwavelength Optical Crossconnect for Parallel-Processing Computers," Electronics Letters, January 1988, Vol. 24, No. 2, pp.119-120.

[6] C. G. Atkins, D. Cotter, D. W. Smith and R. Wyatt, "Application of Brilliouin Amplification in Coherent Optical transmission," Electronics Letters, Vol. 22, No. 10, May 8, 1986, pp.556-558.

[7] E. J. Bachus, R. P. Braun, C. Casper, H. M. Foisel, E Grossmann, B. Streble and F. J. Westphal, "Coherent Multicarrier Systems," Journal of Lightwave Technology, Vol. 7, No. 2, february 1989, pp. 375-384.

[8] Ralph Ballart and Yau-Chau Ching, "SONET: Now it's the Standard Optical Network," IEEE Communications Magazine, Vol. 27, No. 3, March 1989, pp. 9-15.

[9] K. E. Batcher, "Sorting Networks and Their Applications," Proceedings of the 1968 Spring Joint Computer Conference, pp. 307-314.

[10] P. C. Becker, J. R. Simpson, and N. K. Dutta, "High-Gain and High-efficiency diode Laser Pumped Fiber Amplifier at $1.56 \mu \mathrm{m}$," IEEE Photonics Technology Letters, Vol. 1, No. 9 September 1989, pp. 267-269.

[11] Trudey E. Bell, "Fiber Optics," IEEE Spectrum, 25th Anniversary Issue, 1988, Vol. 25, No. 11, pp. 97-102.

[12] Rodney J. Bohem, Yau-Chau Ching, C. George Griffith, Frederick A. Saal, "Standardized Fiber Optic Transmission Systems-A Synchronous Optical Network," IEEE Joumal on Selected Areas in Communications, Vol SAC-4, No. 4, June 1986, pp. 1424-1431.

[13] Werner Bux, Felix H. Closs, Karl Kuemmerle, Heinz J. Keller and Hans R. Mueller, "Architecture and Design of a Reliable Token-Ring Network," IEEE Journal on Selected Areas in Communications, Vol. SAC-1, No. 5, November 1983, pp. 756-765.

[14] William R. Byrne, Toomas A. Kilm, Bruce L. Nelson and Marius D. Soneru, "Broadband ISDN Technology and Architecture," IEEE Network, January 1989, pp. 23-28. 
[15] K. W. Cheung, M. M. Choy and H. Kobrinski, "Electronic Wavelength Tuning Using Acoustooptic Tunable Filter with Broadband Continuous Tuning Range and Narrow Channel Spacing," IEEE Photonics Technology Letters, Vol. 1, No. 2, Feb. 1989, pp. 38-40.

[16] Y. C. Chung, K. J. Pollock, P. J. Fitzgerald, B. Glance, R. W. Tkach, and A. R. Chraplyvy, "WDM Coherent Star Network with Absolute Frequency Reference," Electronics Letters, 13th October 1988, Vol. 24, No. 21, pp. 1313-1314.

[17] Peter Cochrane, and Mike Brain, "Future Optical Fiber Transmission Technology and Networks," IEEE Communications Magazine, Vol. 26, No. 11, November 1988, pp. 45-60.

[18] C. Coquin, H Kobrinski, C. E. Zah, F. K. Shokoohi, C. Caneau, and S. G. Menocal, "Simultaneous Amplification of 20 Channels in a Multiwavelength Distribution System,” IEEE Photonic Technology Letters, Vol. 1, No. 7, July 1989, pp. 176-178.

[19] George E. Daddis, Jr. and H. C. Torng, “A Taxonomy of Broadband Integrated Switching Architectures,” IEEE Communications Magazine, Vol. 27, No. 5, May 1989, pp.32-42.

[20] T. E. Darcie, "Subcarrier Multiplexing for Multiple-Acess Lightwave Networks," Journal of Lightwave Technology, Vol. LT-5, No. 8, August 1987, pp. 1103-1110.

[21] T. E. Darcie, P. P. Iannone, B. L. Kasper, J. R. Talman, C. A. Burris and T. A. Baker, "Wide-Band Lightwave Distribution System Using Subcarrier Multiplexing," Journal of Lightwave Technology, Vol. 7, No. 6, June 1989, pp. 997-1005.

[22] Niloy K. Dutta, "III-V Device Technologies for Lightwave Applications," AT\&T Technical Journal, January/February 1989, pp. 5-18.

[23] N. Edagawa, K. Mochizuki, and H. Wakabayshi, “1.2 gbit/s $218 \mathrm{~km}$ Transmission experiment using Inline Er-Doped Optical Riber Amplifier,” Electronic Letters, Vol. 25, No. 5, March 2, 1989, pp. 363-365.

[24] Martin Eisenberg and Nader Mehravari, "Performace of the Multichannel Multihop Lightwave Network Under Nonuniform Traffic," IEEE Journal on Selected Areas in Communications, Vol. 6, No. 7, August 1984, pp. 1063-1078.

[25] Joseph D. Evankow, Jr. and Richard A. Thompson, "Photonic Switching Modules Designed with Laser Diode Amplifiers," IEEE Journal on Slected Areas in Communications, Vol. 6, No. 7, August 1988, pp. 1087-1095.

[26] Yves Charles Faroudja, "NTSC and Beyond," IEEE Transactions on Consumer Electronics, Vol. 34, No. 1, February 1988, pp. 166-178.

[27] ANSI X3.139-1987, American National Standard for Information Systems - Fiber Distributed Data Interface (FDDI) - Token Ring Media Access Control (MAC), American National Standards Institute, New York, 1987. 
[28] ANSI X3.148-1987, American National Standard for Information Systems - Fiber Distributed Data Interface (FDDI) - Token Ring Physical Layer Protocol (PHY),

American National Standards Institute, New York, 1988.

[29] dpANS X3.166-198X, draft proposed American National Standard for Information Systems - FDDI Physical Layer Medium Dependent (PMD), X3T9.5/84-48 Rev. 9, available from Global Engineering Documents, Irvine, CA, March 1, 1989.

[30] Stephen R. Forrest, “Optical Detectors: Three Contenders," IEEE Spectrum, May 1986, pp. 76-84.

[31] Gerald J. Foschini and Giovanni Vannucci, "Using Spread-Spectrum in a High Capacity Fiber-Optic Local Network," Journal of Lightwave Technology, Vol. 6, No. 3, March 1988, pp. 370-379.

[32] Hugo Gaggioni and Didier Le Gall, "Video Transmission and Coding for Broadband ISDN," IEEE Transactions on Consumer Electronics, Vol. 34, No. 1, February 1988, pp.16-34.

[33] Jerry Gechter and Peter O'Reilly, "Conceptual Issues for ATM," IEEE Network, January 1989, pp. 14-16.

[34] B. S. Glance, J. stone, K. J. Pollock, P. J. Fitzgerald, C. A. Burris, Jr., B. L. Kasper and L. W. Stulz, "Densely Spaced Cohereant Star Network With Optical Signals confined to Equally Spaced Frequencies," Journal of Lightwave Technology, Vol. 6, No. 11, November 1988, pp. 1770.

[35] B. Glance, T. L. Koch, O Scaramucci, K. C. Reichamnn, U. Koren and C. A. Burrua, "Densely Spaced FDN using Coherent Optical Star Network Using Monolythic Widely Frequency-Tunable Lasers," Electronics Letters, 11th May 1989, Vol. 25, No. 10, pp.672-673.

[36] Richard Gross, Robert Olshansky and Paul Hill, "20 Channel Coherent FSK System Using Subcarrier Multiplexing," IEEE Photonics Technology Letters, Vol. 1, No. 8, August, 1989, pp. 224-226.

[37] Rainer Handel, "Evolution of ISDN Towards Broadband ISDN," IEEE Network, January 1989, pp. 7-13.

[38] Jim Hamstra, "FDDI Integrity Goals," presentation to the FDDI SMT Working Group, Feb. 1989.

[39] Mitsutoshi Hatori and Yoshiro Nakamura, "1125/60 HDTV Studio Standard Intended to Be a worldwide Unified HDTV Standard" IEEE Transactions on Broadcasting, Vol. 35, No. 3, September 1989, pp.270-278.

[40] Jeff Hecht, "Optical Amplifiers: A Horse race with Promise," Lasers \& Optionics, Vol. 8, No. 7, July 1989, pp. 14-22.

[41] Christine F. Hemrick, Robert W. Klessig and Josephine M. McRoberts, "Switched Multimegabit Data Service and Early Availability via MAN Technology," IEEE Communications Magazine, April 1988, Vol. 26, No. 4, pp. 9-14. 
[42] Paul S. Henry, "Lightwave Primer," IEEE Journal of Quantum Electronics, Vol. QE-21, No. 12, December 1985, pp.1862-1879.

[43] H. Scott Hinton, "Architectural Considerations for Photonic Switching Networks," IEEE Journal on Selected Areas in Communications, Vol. 6, No. 7, August 1988, pp. 1209-1226.

[44] Robert Hopkins, "Advanced Television Systems," IEEE Transactions on Consumer Electronics, Vol. 34, No. 1 February 1988, pp. 1-15.

[45] Robert Hopkins and Kenneth P. Davies, "Development of HDTV Emission Systems in North America," IEEE Transactions on Broadcasting, Vol. 35, No. 3, September 1989, pp. 259-269.

[46] Andrew Hopper and Robin C. Williamson, "Design and Use of an Integrated Cambridge Ring," IEEE Journal on Selected Areas in Communications, Vol. SAC-1, No. 5, November 1983, pp. 775-784.

[47] Daniel E. Huber, Walter Steinlin and Peter J. Wild, "SILK: An Implementation of a Buffer Insertion Ring," IEEE Journal on Selected Areas in Communications, Vol. SAC-1, No. 5, November 1983, pp.766-775.

[48] A. Huang and S. Knauer, "Starlite: A Wideband Digital Switch," in Proceedings IEEE Globecom '84 , pp. 121-125.

[49] Joseph Y. Hui,“Network, Transport, and Switching Integration for Broadband Communications," IEEE Network, March 1989, pp. 40-51.

[50] D. J. Hunkin, G. R. Hill and W. A. Stallard, "Frequency Locking of External Cavity Semiconductor Lasers Using an Optical Comb Generator," Electronics Letters, Vol. 22, No. 7, April 1986, pp. 388-390.

[51] Michael A. Isnardi, Terrance R. Smith and Barbara J. Roeder, "Decoding Issues in the ACTV System," IEEE Transactions on Consumer Electronics, Vol. 34, No. 1, February 1988, pp.111-120.

[52] Tetsuhiko Ikegami and Hitoshi Kawaguchi, "Semiconductor Devices in Photonic Switching," IEEE Journal on Selected Areas in Communications, Vol. 6, No. 7, August 1988, pp. 1131-1140.

[53] G. R. Joyce, V. Lanzisera and R. Olshansky, "Improved Sensitivity of 60 Video Channel FM-SCM Receiver with Semiconductor Optical Amplifier," Electronics Letters, 13th April 1989, Vol. 25, No. 8, pp. 499-500.

[54] Tatsuya Kimura, "Coherent Optical Fiber Transmission," Journal of Lightwave Technology, Vol. LT-5, No. 4, April 1987, pp. 414-428.

[55] I. P. Kaminow, P.P. Iannone, J. Stone and W. L. Stulz, "FDMA-FSK Star Network with a Tunable Optical Filter Demultiplexer," Journal of Lightwave Technology, Vol. 6, No. 9, September 1988, pp. 1406-1414. 
[56] Ivan P. Kaminow, "Non-coherent Photonic Frequency-Multiplexed Access Networks," IEEE Network, March 1989, pp. 4-12.

[57] I. P. Kaminow, P. P. Iannone, J. Stone, and L. W. Stulz, “A Tunable Vernier Fiber Fabry-Perot filter for FDM Demultiplexing and Detection," IEEE Photonics Technology Letters, Vol. 1, No. 1, Jan.1989, pp. 24-26.

[58] H. Kobrinski, R. M. Bulley, M. S. Goodman, M. P. Vecchi C. A. Brackett, L. Curtis and J. L. Gimlett, "Demonstration of a High Capacity In the Lambdanet Architecture: A Multiwavelength Optical Network," Electronics Letters, July 1987, Vol. 23, No. 16 , pp. 824-826.

[59] Stephen K. Korotky, "Ti:LiNbO 3 Waveguides Support High-Speed Modulation and Switching," Laser Focus World, June 1989 pp. 151-158.

[60] Tony T. Lee, "Nonblocking copy Networks for Multicast Packet Switching," IEEE Journal on Selected Areas in Communications, Vol. 6, No. 9, December 1988, pp.14551467.

[61] Richard Li and Richard A. Linke, "Multigigabit-Per-Second Lightwave Systems ReSearch for Long-Haul Applications," IEEE Communications Magazine, Vol. 26, No. 4, April 1988, pp. 29-35.

[62] Richard A. Linke and Alan H. Gnauck, "High-Capacity Coherent Lightwave Systems," Journal of Lightwave Technology, Vol. 6, No. 11, November 1988, pp. 1750-1769.

[63] Richard A. Linke, "Frequency Division Multiplexed Optical Networks Using Optical Hetrodyne Detection,” IEEE Network, March 1989, pp. 13-20.

[64] R. A. Linke, K. C. Reichmann, T. L. Kochand U. Koren, "Full-Duplex Optical transmission Using Self-Heterodyne Laser Receivers," IEEE Photonics Technology Letters, Vol. 1, No. 9, September 1989, pp. 278-280.

[65] R. Ian MacDonald, "Termanology for Photonic Matrix Switches," IEEE Journal on Selected Areas in Communications, Vol. 6, No. 7, August 1988, pp. 1141-1151.

[66] D. J. Malyon, R. C. Steele, M. J. Creaner, M. C. Brain and W. Stallard, "Coherent Optical Transmission at $565 \mathrm{Mbit} / \mathrm{s}$ Through Five Cascaded Photonic Amplifiers," Electronics Letters, Vol. 25, No. 5, March 2, 1989, pp. 354-356.

[67] R. M. Metcalf and D. R. Boggs, "Ethernet: Distributed Packet Switching for Local Area Networks," Communications of the ACM, Vol. 19, No. 7, July 1976, pp. 395 404.

[68] A. Myles, "DQDB Simulation and MAC Protocol Analysis," Electronic Letters, 27th April 1989, Vol. 25, No. 9, pp. 616-617.

[69] Hitomi Murakami, Hidel Hashimito and Yoshinori Hatori, "Quality of Band-Compressed TV Services,” IEEE Communications, October 1988, pp. 61-69.

[70] Richard L. Nickelson, "The Evolution of HDTV in the Work of the CCIR," IEEE Transactions on Broadcasting, Vol. 35, no 3. Setember 1989, pp. 250-258. 
[71] Eric Nussbaum, "Communications Network Needs and Technologies - A place for Photonic Switching?", IEEE Journal on Selected Areas in Communications, Vol. 6, No. 7, August 1988, pp. 1036-1043.

[72] M. G. Oberg, N. A. Olsson, K. A. Koszi and G. J. Przybylek, "313km Transmission Experiment at $1 \mathrm{Gbit} / \mathrm{s}$ Using Optical Amplifiers and a Low Chirp Laser," Electronics Letters, January 7 1988, Vol. 24, No. 1, pp.38-39.

[73] T. Okoshi and K. Kikuchi, "Frequency Stabilization of Semiconductor Lasers for Heterodyne-Type Optical Communication Systems," Electronic Letters, Vol. 16, no 5, Feb 28, 1980, pp. 179-181.

[74] R. Olshansky and V. A. Lanzisera, "60-Channel FM video Subcarrier Multiplexed Optical Communication System," Electronics Letters, 22nd October 1987, Vol. 23, No. 23, pp. 1196-1197.

[75] R. Olshansky, V. A. Lanzisera and Paul M. Hill, "Subcarrier Multiplexed Lightwave Systems for Broadband Distribution," Journal of Lightwave Technology, Vol. 7, No. 9, September 1989, pp. 1329-1341.

[76] Raymond L. Pickholtz, Donald Schilling, Laurence B. Milstein, "Theory of SpreadSpectrum Communications-A Tutorial," IEEE Transactions on communications, vol COM-30, No. 5, May 1982, pp. 855-884.

[77] Paul R. Prucnal, Mario A Santoro, Sanjay K. Sehgal, "Ultrafast All-Optical Synchronous Multiple Access fiber Networks," IEEE Journal on Selected Areas in Communications, Vol. SAC-4, No. 9, December 1986, pp. 1484-1493.

[78] D. Raychaudhuri and K. Joseph, "Channel Access Protocols for Ku-band VSAT Networks; A Compartative Evaluation," IEEE Communications, Vol. 26, No. 5, May 1988, pp. 34-35.

[79] Michael J. Rider, "Prorocols for ATM Access Networks," IEEE Network, January 1989, pp. 17-22.

[80] P. A. Roshner, S. C. Fenning, M. J. Ramsay and F. V. C. Mendis, "Broadband Video Distribution Over Passive Optical Networking Using Subcarrier Multiplexing Techniques," Electronics Letters, 19th January 1989, Vol. 25, No. 2, pp.115-117.

[81] Tadashi Saitoh and Takaaki Mukai, "Recent Progress in Semiconductor Laser Amplifiers," Journal of Lightwave Technology, Vol. 6, No. 11, November 1988, pp. 1656-1664.

[82] Jawad A. Salehi, "Emerging Optical Code-division Multiple Access Communications Systems," IEEE Network, March 1989, pp 31-39.

[83] Robert A. Scholtz, "The Origins of Spread-Spectrum Communications," IEEE Transactions on Spread-Spectrum Communications, Vol. COM-30, No. 5, May 1982, pp.822854. 
[84] A. S. Siddiqui and S. Andronikidis, "Transfer Characteristics of Brillouin Fibre Amplifiers for use in Self-Homodyne Coherent Optical Transmission Systems," Electronics Letters, Vol. 25, No. 4, Feb. 16, 1989, pp. 264-266.

[85] J. C. Simon, "Polarization characteristics of a traveling wave semiconductor laser amplifiers," Electronics Letters, Vol. 18, No. 11, pp. 240-248, 1983.

[86] "SMPTE tackles HDTV," Broadcasting, February 13, 1989, pp. 82-84.

[87] Armin Sollberger, Arja Heinamaki and Hans Melchior, "Frequency Stabilization of Semiconductor Lasers for Applications in coherent communications Systems," Journal of Lightwave Technology, Vol. LT-5, No. 4, April a987, 485-491.

[88] I. W. Stanley, G. R. Hill and D. W. Smith "The Application of Coherent Optical Techniques to Wide-Band Networks," Journal of Lightwave Technology, Vol. LT-5, No. 4, April 1987, pp m439-451.

[89] Ludwig Stenger, "Digital Coding of TV Signals for ISDN-B Applications," IEEE Journal on Selected Areas in Communications, Vol. SAC-4, No. 4, July 1986, pp 514-528.

[90] J. R. Stern, J. W. Ballance, D. W. Faulkner, S. Hornung and D. B. Payne, "Passive Optical Local Networks for Telephony Applications and Beyond," Electronics Letters, 19th November 1987, vol.23, No. 24, pp 1255-1257.

[91] Harish R. D. Sunak, Steven P. Bastein, "Characteristics of Dispersion-Flattened Depressed-Cladding Single-Mode Flouride Fibers from 1.55 to 3.1 um," IEEE Photonics Technology Letters, Vol. 1, No. 8, august 1989, pp.224-247.

[92] Larry Thorpe, "HDTV: It's the Size that Counts," Video Review, August 1989, p. 92.

[93] Marc Thompson, "Designing Video Circuits," ESD: The Electronic System Design Magazine, October 1988 pp. 67-74 and November 1988, pp. 75-79.

[94] R. W. Tkach, A. R. Chraplyvy and R. M. Derosier, "Performance of a WDM Network Based on Stimulated Brillouin Scattering," IEEE Photonics Technology Letters, Vol. 1, No. 5, May 1989, pp.111-113.

[95] Hirmou Toba, Kyo Inoue and Kiuoshi Nosu, "A Conceptual Design on Optical Frequency-Division-Multiplexing Distribution Systems with Optical Tunable Filters," IEEE Journal on Selected Areas in Communications, Vol. SAC-4, No. 9, December 1986, pp. 1458-1781.

[96] H. Toba, K. Oda and K. Nosu, "16-channel Optical FDM Distribution /Transmission Experiment Utilizing Multichannel Frequency Stabiliser and Waveguide Frequency Selection," Electronics Letters, 27th April 1989, Vol. 25, No. 9, pp.574-575.

[97] W. J. Tomlinson, and Roger H. Stolen, "Nonlinear Phenomena in Optical Fibers," IEEE Communications Magazine, Vol. 26, No. 4, April 1988, pp.36-44.

[98] Arpand G. Toth, Mikhail Tsinberg and Charles W. Rhodes, "NTSC Compatible High Definition Television Emission System," IEEE Transactions on Consumer Electronics, Vol. 34, No. 1 February, 1988, pp. 40-47. 
[99] Jonathan S. Turner, "Design of an Integrated Services Packet Network," IEEE Journal on Selected Areas in Communications, Vol. SAC-4, No. 8, November 1986, pp. 13731380.

[100] Jonathan S. Turner, "Design of a Broadcast Packet Network," in Propceedings of Infocom '86, pp. 667-675.

[101] Jonathan S. Turner, "New directions in communicatns (or Which Way to the Information Age?)" IEEE Communications Magazine, Vol. 24, No. 10, 1986, pp. 8-15.

[102] Hitoshi Uematsu and Ryuichi Watanabe, "Architecture of a Packet Switch Based on Banyan Switching Network with Feedback Loops," Journal on Selected Areas in Communications, Vol. 6, No. 9, December 1988, pp. 1521-1527.

[103] T. Valovic, "Metropolitan Area Networks: a Status Report," Telecommunications, July 1989, pp. 25-32.

[104] Giovanni Vannucci, “Combining Frequency-Division and Code Division Multiplexing in a High-Capacity Optical Network," IEEE Network, March 1989, pp. 21-30.

[105] Jan P. Vorstermans and Andre P. De Vleeschouwer, "Layered ATM Ststems and Architectural Concepts for Suscribers' Premises Networks," IEEEE Journal on Selected Areas in Communications, Vol. 6, No. 9, december 1988, pp. 1545-1555.

[106] Stuart S. Wagner and Haim Kobrinski, "WDM Applications in Broadband Tellecummunication Networks," IEEE Communications Magazine, Vol 27, No. 3, March 1989, pp. 22-29.

[107] Nick Wainright and Andrew Myles, “ A comparison of the Delay Characteristics of FDDI and IEEE 802.6 MAC Layer Protocols," Hewlett Packard Laboratories Information Systems Centre, Bristol, England, March 13, 1989, presented at the 3rd IEEE MAN Workshop in San Diego.

[108] W. I. Way, R. S. Wolf and M. Krain, "A 1.3-um 35 km Fiber-Optic Microwave Multicarier Transmission System for Satellite Earth Stations," Joumal of Lightwave Technology, Vol. LT-5, No. 9, September 1987, pp. 1325-1332.

[109] R. Welter and L. G. Kazovoski, "150 Mbit/s Phase Diversity ASK Homodyne Receiver with a Linewidth/Bit Ratio of 0.5," Electronics Letters, February 18, 1988, Vol. 24, No. 4, pp. 199-201.

[110] M. V. Wilkes and D. J. Wheeler, "The Cambridge Digital Communication Ring," presented at Local Area Communications Network Symposium, Boston, MA., May 1979, sponsored by the National Bureau of Standards.

[111] T. J. Whitley, "Laser Diode Pumped Operation of $\mathrm{Er}^{+3}$-Doped Fiber Amplifier," Electronics Letters, Vol. 24, No. 25, Dec. 8, 1988, pp.1537-1539.

[112] C. J. Wolejsza, D. Taylor, M. Grossman and W. P. Osborne, "Multiple Access Protocols for Data Communications via VSAT Networks," IEEE Communications Magazine, July 1987, Vol. 25, No. 7, 1987, pp. 30-37. 
[113] C. L. Wu and T. Y. Feng, "On a Class of Multistage Interconnection Networks," IEEE Transactions on Computing, Vol. C-29, August 1980, pp. 694-702.

[114] R. Wyatt and J. W. Devlin, "10kHz $1.5 \mu \mathrm{m}$ InGaAsP External Cavity Laser with 55 nm Tuning Range,” Electronics Letters, Vol. 19., pp. 110-112, 1983.

[115] Yoshio Yasumoto, Sadashi Kageyama, Syuhji Inouye, Hideyo Uwabata and Yoshio Abe, "A Wide Aspect Ratio Television System with Full NTSC Compatibiliity," IEEE Transactions on consumer Electronics, Vol. 34, No. 1 February 1988, pp.121127.

[116] Project - 802, Local and Metropolitan area Networks, Proposed Standard for: "Distributed Queue Dual Bus (DQDB) Metropolitan Area Network," 23 June 1989.

[117] Harold S. Stone, "Parallel Processing with a Perfect Shuffle," IEEE Transactions on Computers, Vol. C-20, No. 2, February 1971, pp. 153-161.

[118] James M. McCarthy, International Competitiveness Study of: The Fiber Optics Industry, U. S. Department of Commerce, September 1988.

[119] Kai Y. Eng, “A Photonic Knockout Switch for High-Speed Packet Networks," IEEE Journal on Selected Areas in Communications, Vol. 6, No. 7 August 1988, pp. 1107. 1116. 


\section{Appendix A \\ List of Acronyms}

$\begin{array}{ll}\text { AM } & \text { Amplitude Modulation } \\ \text { APD } & \text { Avalanche PhotoDiode } \\ \text { ARPA } & \text { Advanced Research Projects Agency } \\ \text { ASK } & \text { Amplitude Shift Keying } \\ \text { ATM } & \text { Asynchronous Transfer Mode } \\ \text { B-ISDN } & \text { Broadband Integrated Services Digital Network } \\ \text { BER } & \text { Bit Error Rate } \\ \text { CATV } & \text { Cable Access TeleVision } \\ \text { CCIR } & \text { International Radio Consultative Committee } \\ \text { CDMA } & \text { Code-Division Multiple Access } \\ \text { CSMA } & \text { Carrier Sense Multiple Access } \\ \text { CSMA/CD } & \text { Carrier Sense Multiple Access with Collision Detection } \\ \text { DBR } & \text { Distributed Bragg Reflector } \\ \text { DFB } & \text { Distributed FeedBack laser } \\ \text { DQDB } & \text { Dual Queue, Dual Bus } \\ \text { ECL } & \text { External Cavity Laser } \\ \text { FCC } & \text { Federal Communications Commission } \\ \text { FDDI } & \text { Fiber distributed Data Interface } \\ \text { FDM } & \text { Frequency Division Multiplexing } \\ \text { FDMA } & \text { Frequency Division Multiple Access } \\ \text { FPA } & \text { Fabry-Perot Amplifier } \\ \text { FSK } & \text { Frequency Shift Keying } \\ \text { FSR } & \text { Free Spectral Range } \\ \text { HDTV } & \text { High Definition TeleVision } \\ \text { IC } & \text { Integrated Circuit } \\ \text { IEEE } & \text { Institute of Electrical and Electronics Engineers } \\ \text { ISDN } & \text { Integrated Services Digital Network } \\ \text { LAN } & \text { local Area Network } \\ \text { LD } & \text { Laser Diode } \\ \text { LED } & \text { Light Emitting Diode } \\ \text { MAC } & \text { Medium Access Control } \\ \text { MAN } & \text { Metropolitan Area Network } \\ \text { NHK } & \text { Japan Broadcasting Company } \\ \text { NTSC } & \text { National Television Standards Committee } \\ \text { OSI } & \text { Open systems Integration } \\ \text { PC } & \text { Personal Computer } \\ \text { PDU } & \text { Protocol Data Unit } \\ \text { PIN } & \text { P-type, Intrinsic, N-type } \\ \text { PON } & \text { Passive Optical Network } \\ \text { POTS } & \text { Plain Old Telephone Service } \\ \text { PSK } & \text { Phase Shift Keying } \\ \text { RBG } & \text { Red Blue Green } \\ \text { SCM } & \text { Subcarrier Division Multiplexing } \\ \text { SLA } & \text { Semiconductor Laser Amplifier } \\ \text { SMDS } & \text { Switched Multimegabit Data Service } \\ \text { SMPTE } & \text { Society of Motion Picture and Television Engineers } \\ \text { SNR } & \text { Signal to Noise Ratio } \\ & \\ & \end{array}$


SONET Synchronous Optical NETwork

TDM Time Division Multiplexing

TDMA Time Division Multiple Access

TWA Traveling Wave Amplifier

VLSI Very Large Scale Integration

VSAT Very Small Aperture saTellite

WAN Wide Area Network

WDM Wavelength Division Multiplexing 


\begin{tabular}{|c|c|c|}
\hline \multirow[t]{3}{*}{$\begin{array}{l}\text { NIST-114A } \\
\text { (REV. 3-09) }\end{array}$} & \multirow{3}{*}{$\begin{array}{l}\text { U.S. DEPARTMENT OF COMMERCE } \\
\text { NATIONAL INSTITUTE OF STANDARDS AND TECHNOLOGY } \\
\text { BIBLIOGRAPHIC DATA SHEET }\end{array}$} & $\begin{array}{l}\text { 9. PUDUCATION OR REPOAT MUMDEA } \\
\text { NISTIR } 90-4240\end{array}$ \\
\hline & & 2 PERFORMINO OR CAMIZATION REPOAT NUMEER \\
\hline & & $\begin{array}{r}\begin{array}{r}\text { PULUCATION DATE } \\
\text { MARCH } 1990\end{array} \\
\end{array}$ \\
\hline
\end{tabular}

4. TITE AND SUCTITL

Architectures for Future Multigigabit Lightwave Networks

5. AUTHOR(S)

William E. Burr

6. PERFORMINO OACAMLZATION (IF JOINT OA OTHER THAN NIST, SEE IMSTRUCTIONS)

U.8. DEPAMTMENT OF COMMERCE

MATIONAL MSTIUUTE OF STANOARDS ANO TECHNOLOCY

carthenseuna, Mo 2009

7. CONTMCT/ORAT MUMSEA

8. TME OF REPORT ANO PERIOD COVERED

9. SPONSORLO ORCWIZATION MAME AMD COMPLETE ADDRESS (BTRET, CTY, STATE, ZI)

10. SUPPLEMTTARY NOTES

DOCUMENT DESCRIEES A COMPUTER PROGRAM; SF-185, FIPS SOFTWARE SUMMANV, RS ATTACHED.

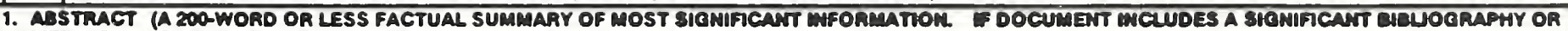
ITERATURE SURVEY, MENTION TT HERE.)

Architectures for future fiber optic networks for volce, video and data applications are considered. Broadband ISDN (B-ISDN) w1ll be introduced for these applications sometime after 1995. B-ISDN w111 be based on fast switching of rather smal1 (48-byte) packets. The packet switches themselves w111.probably be electronic and will take advantage of the ability of semiconductor electronic technology to bulld very complex, regular, repetitive structures. Packet switching provides a flexible, efficlent means of sharing bandwidth limited communications resources. Developing fiber optic technology, however, offers another revolutionary alternative, the Passive Optical Network (PON), based on the realization that the Inherent bandwidth of the fiber 18 Immense and using optical frequency division multiplexing to exploit this. The PON has significant advantages over centralized packet switching in some applications (particularly video distribution), is simpler, facilitates the addition of new services, can be begun and extended in small increments, and requires much less initial investment in the network. However, the PON depends upon the commercial development of several components, such as tunable lasers and coherent receivers, which have so far been proven only in laboratories.

12. KEY WORDS (6 TO 12 ENTAIES; ALPHABETICAL ORDER; CAPTTALEE ONLY PROPEA MAMES; AHD SEPARATE KEY WORDS OY SEMICOLONS) B-ISDN, fast packet switching, flber optic networks, frequency division optical networks, integrated service networks, local area networks, metropolitan area networks, multiglgabit networks, passive optical networks, wide area networks

FOR OFFICLAL DISTAIBUTION. DO MOT RELEASE TO MATIOMAL TECHNICAL INFORMATION SEAVCE (NTIS).

14. NUMBER OF PRINTED PAQES

68

15. PRICE A04 


